\title{
Intermediate Energy Nuclear Physics Program
}

\author{
Final Technical Report \\ August 15, 2004 - August 142010 \\ James A. Dunne \\ Department of Physics and Astronomy \\ Mississippi State University \\ Mississippi State, MS 39762
}

June 22, 2012

Prepared for the U.S. Department of Energy

Under Grant Number DE-FG02-04ER41330 


\section{Contents}

1 Research Report 1

1.1 Research Summary . . . . . . . . . . . . . . . . . . . . . . . . 1

1.2 Sub-threshold $J / \psi$ Production . . . . . . . . . . . . . . . . . . 2

1.2 .1 Background . . . . . . . . . . . . . . . . . . . 2

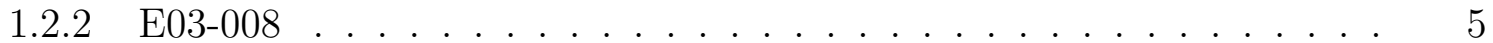

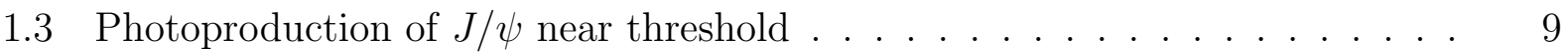

1.3.1 Proposed Measurement . . . . . . . . . . . . . . . . . . . . 11

1.4 Qweak Target Work . . . . . . . . . . . . . . . . . . . . . . . . . 12

1.4.1 Background to Qweak Measurement . . . . . . . . . . . . . . 12

1.4 .2 Introduction to MSU Target Work . . . . . . . . . . . . . . 15

1.4 .3 Cell Design . . . . . . . . . . . . . . . . . . . . . . . . . . 15

1.4.4 High Power Heater . . . . . . . . . . . . . . . . . . . . 16

1.4.5 Motion Mechanism . . . . . . . . . . . . . . . . . . 17

1.4 .6 Vertical Travel . . . . . . . . . . . . . . . . . . . . . 20

1.4 .7 Alignment . . . . . . . . . . . . . . . . . . . . . . . 20

1.4 .8 Heat Load . . . . . . . . . . . . . . . . . . . . . . . . . . . . . . . 21

1.5 Areal Densities for Hall C Experiments during 2005 running . . . . . . . . . 21

1.6 Spin Structure of Nucleons . . . . . . . . . . . . . . . . . . . . . . 22

1.6.1 Spin Asymmetries on the Nucleon Experiment: SANE . . . . . . . 22

2 Publications and Reports $\quad 24$

3 Talks Presented at Professional Meetings $\quad 25$

4 Personnel and Collaborators $\quad \mathbf{2 6}$

4.1 Principal Collaborators in the Reporting Period . . . . . . . . . . . . 27

$\begin{array}{llr}5 & \text { Bibliography } & 29\end{array}$ 


\section{Research Report}

\subsection{Research Summary}

The originally proposed and funded research activities followed two major areas of study: semileptonic probes of the hadronic neutral current and charm production. The charm production work revolved around the Jefferson Lab experiment E03-008, "Sub-threshold $J / \psi$ Photoprouction", which ran in late 2004. The PI was a co-spokesperson for the experiment. For the three year renewal proposal starting in 2007, the scope and size of the research project changed and increased. In addition to the parity violating studies, the PI had well defined lead roles in a series experiments nucleon spin-structure functions (SANE [32]; SemiSANE [2], and E07-011 [3]). This was a return to the PI's roots, as he did his Ph.D thesis on the first measurement of the neutron spin structure extracted from a helium target (SLAC experiment E-142 [4]). The experimental setup for these spin structure measurements planned to utilize a large solid angle (Big) Electron Telescope Array (BETA) primarily consisting of a large Čerenkov detector plus an electromagnetic shower counter. Both SANE and E07011 planned on using the BETA detectors and the high momentum spectrometer (HMS) for inclusive measurements, while Semi-SANE planned on using the two in coincidence mode. SANE's focus was on the extraction of the high $x$ part of the spin structure functions of the proton. Semi-SANE would have measured the spin asymmetries in the semi-inclusive deep-inelastic $\vec{p}\left(e, e^{\prime}, h\right) X$ and $\vec{d}\left(e, e^{\prime}, h\right) X$ reactions $\left(h=\pi^{+}, \pi^{-}, K^{+}\right.$, and $\left.K^{-}\right)$on longitudinally polarized $\mathrm{NH}_{3}, \mathrm{ND}_{3}$, and LiD targets. The g1d experiment (E07-011) would have overlapped with Semi-SANE's deuteron runs plus some additional kinematic settings which will allow the extraction of deuteron spin-structure function $g_{1}^{d} / F_{1}^{d}$. The principle goal was to provide the low $Q^{2}$ anchor points for NLO pQCD plus higher twist fits to $g_{1}^{d} / F_{1}^{d}$, which is particularly sensitive to the polarized gluon density of the nucleon $\Delta G(x)$ and the sum of up and down quark polarizations. Unfortunately, only the SANE experiment ran during this grant period. The PI's graduate student, Luwani Ndukum, is one of the $\mathrm{PhD}$ thesis students analyzing the SANE data.

The research area of semileptonic probes of the hadronic neutral current revolved around the Jefferson Lab experiment E08-016, "The $Q_{\text {weak }}$ Experiment: A Search for New Physics at the TeV Scale Via a Measurement of the Proton's Weak Charge" of which the PI is an active collaborator. E08-016 will provide a unique opportunity to perform a precision low energy test of the Standard Model and look for evidence of physics beyond the Standard Model. This will be possible by measuring the proton's weak charge, $Q_{w}^{p}=1-4 \sin ^{2} \theta_{w}$, at low $Q^{2}$. This program of study has only recently been possible due to the strong, on-going parity-violating (PV) electron scattering program developed at Jefferson Lab. These PV experiments are providing the data on the form factors used to determine the contributions of hadronic structure to the $Q_{w}$ measurement, thus eliminating the need for theoretical nucleon structure calculations. The experiment was put on the floor in Jefferson Lab's Hall C in 2010, and started commissioning after the completion of this grant. Hence, the majority of $Q_{w}$ worked focused on the PI's role in the design, construction, and commissioning of the high power cryogenic liquid hydrogen target. The PI is a vital part of the $Q_{w}$ target group consisting of Jefferson Lab staff, the University of New Hampshire group, and Mississippi 
State University (MSU). The PI's graduate student, Adesh Subedi, is one of the PhD thesis students analyzing the $Q_{w}$ data.

The proposed major research areas will provide an improved understanding of the nucleon and has the potential to look beyond the Standard Model, which were two of the five primary scientific goals for the field during the coming decade as listed by the DOE/NSF Nuclear Science Advisory Committee (NSAC) Long Range Plan [5]. While the charm program addresses directly and indirectly two more of the five questions that NSAC feels will drive nuclear science in the near future.

Regarding charm production, the PI was a co-spokesperson on the Thomas Jefferson National Accelerator Facility (Jefferson Lab) experiment, E03-008 [6], "Sub-threshold $J / \psi$ Photoproduction", which ran during the end of 2004. This experiment was an exploratory measurement which attempted to shed light on the production mechanism of $J / \psi$ mesons in dense nuclear environments due to short range effects, which may be relevant to quark-gluon plasma studies. In other words, the experiment was designed to address how charmonium is produced in kinematics which have an enhanced probability of two or more nucleons in close proximity. The preliminary analysis has ruled out an enhancement of 3-gluon exchange over 2-gluon exchange at threshold. Additionally, this experiment was the beginning of the Jefferson Lab charm program and these data will pave the way for higher energy near-threshold measurements at Jefferson Lab (see section 1.3).

\section{$1.2 \quad$ Sub-threshold $J / \psi$ Production}

\subsubsection{Background}

There is currently very little known about sub-threshold $J / \psi$ photoproduction, either theoretically or experimentally. Theoretical estimates that have been made to date show several order-of-magnitude variations. The goal of experiment E03-008 was to study the production mechanisms in the extreme conditions of high density (short distance scales) that may be relevant in heavy ion collisions where $J / \psi$ suppression is one signature of the formation of a quark-gluon plasma. These conditions are ensured by using a photon beam energy well below photoproduction threshold on a free nucleon. This study is complementary to the studies on nuclei at higher energies, which are more focused on the $J / \psi$-nucleon interaction.

In the single nucleon picture and with beam energies less than $8.2 \mathrm{GeV}$, the nucleon momentum must be pointing anti-parallel to the photon direction for the invariant mass of the photon-nucleon system $s$ to be above the threshold value of $s_{t h}=\left(m+M_{j}\right)^{2}=16.3 \mathrm{GeV}^{2}$, where $m$ is the nucleon mass and $M_{j}$ is the $J / \psi$ mass. The minimum nucleon momentum to reach $s_{t h}$ increases as the photon energy decreases, as shown in Fig. 1a. It is generally thought that the region where multi-nucleon correlations, hidden color configurations, and other short-range effects play a significant role is for momenta beyond $350 \mathrm{MeV}$. This corresponds to a photon of energy of $6 \mathrm{GeV}$, well-matched to the present capabilities of JLab. At lower energies, count rates become prohibitively low, while at higher energies, the Fermisurface contributes most of the cross section, and essentially the reaction processes on a nearly free nucleon are probed. This important part of the program can best be done with 
photon energies of 8 to $11 \mathrm{GeV}$ in the future JLab upgrade [7] (discussed later in section 1.3).
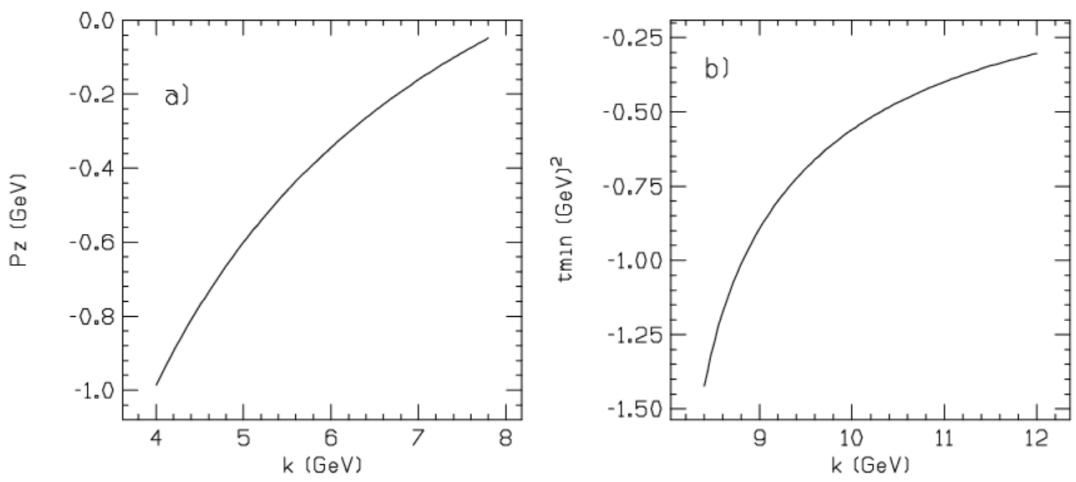

Figure 1: a) The minimum $z$-component of single nucleon momentum (where positive $z$ is along the photon axis) needed for $J / \psi$ photoproduction from a nucleus, as a function of photon energy. b) $t_{\min }$ versus photon energy for photoproduction from a free nucleon.

The high nucleon momentum region is where multi-nucleon correlations are known to be important, and where high density fluctuations could significantly enhance the yield compared to a simple Fermi-smearing model. In the hard-scattering picture [8], this could correspond to strong contributions of higher twist 3-gluon exchange, compared to the minimal 2-gluon exchange needed to ensure the $J / \psi$ color singlet final state. The influence of intrinsic charm contributions, or hidden color contributions, could potentially increase the cross section by an order of magnitude above the expectation from single quasi-free Fermi-smearing (see Fig. 5 of Ref. [8]). Another possibility to enhance the sub-threshold cross section is a diagram in which two gluons are exchanged to two different nucleons. This type of process could become important in the sub-threshold region, because each of the gluons could have a much lower momentum fraction than if the pair of gluons comes from a single nucleon.

\section{Existing data and models near threshold}

There are no published data on sub-threshold photoproduction, but it is useful to review the existing data just above threshold on a free nucleon, as this provides the baseline for sub-threshold predictions based on simple Fermi-smearing models, as described below. The existing data below $20 \mathrm{GeV}$ come from Cornell [9] using 9.3 to $11.8 \mathrm{GeV}$ photons, and from SLAC [10] from 13 to $21 \mathrm{GeV}$. These experiments detected lepton pairs from the $J / \psi$ decay to provide relatively background-free measurements. Additional unpublished data from SLAC [11] detected only a single lepton, leading to relatively large background subtractions.

The experiments typically parameterize the data according to $d \sigma / d t=A e^{b t}$. Values of $b$ from the threshold experiments as well as from high energy experiments are shown in Fig. 2. Interestingly, while $b$ has values of about $6 \mathrm{GeV}^{-2}$ at high energy, which are characteristic of diffractive processes, the values drop rapidly near threshold, with Cornell [9] quoting a value of only $1.25 \pm 0.2 \mathrm{GeV}^{-2}$ near $11 \mathrm{GeV}$ photon energy. The Cornell value is more than a factor of two below the SLAC value of $b=2.9 \pm 0.3 \mathrm{GeV}^{-2}$ at $19 \mathrm{GeV}$. As the curves in the figure show, it is difficult to reconcile the two experiments with a smooth fit, assuming the exponential form corresponds to an effective form factor. One way to resolve this is to 
assume that $d \sigma / d t$ scales as a dipole form factor squared of the form $\left(1-t / m_{0}^{2}\right)^{-4}[12]$. A reasonably good fit to all data up to photon energies of $100 \mathrm{GeV}$ can be found with $m_{0}^{2} \approx 1$ $\mathrm{GeV}^{2}$. Since each experiment measured over a limited range of $t$, and $-t_{\text {min }}$ increases near threshold, a natural explanation for the variation of $b$ with photon energy can be found. We find a reasonable fit to the low energy data is given by $d \sigma / d t=2.5 /(1-t)^{4} \mathrm{nb} / \mathrm{GeV}^{2}$, where $t$ is in units of $\mathrm{GeV}^{2}$.

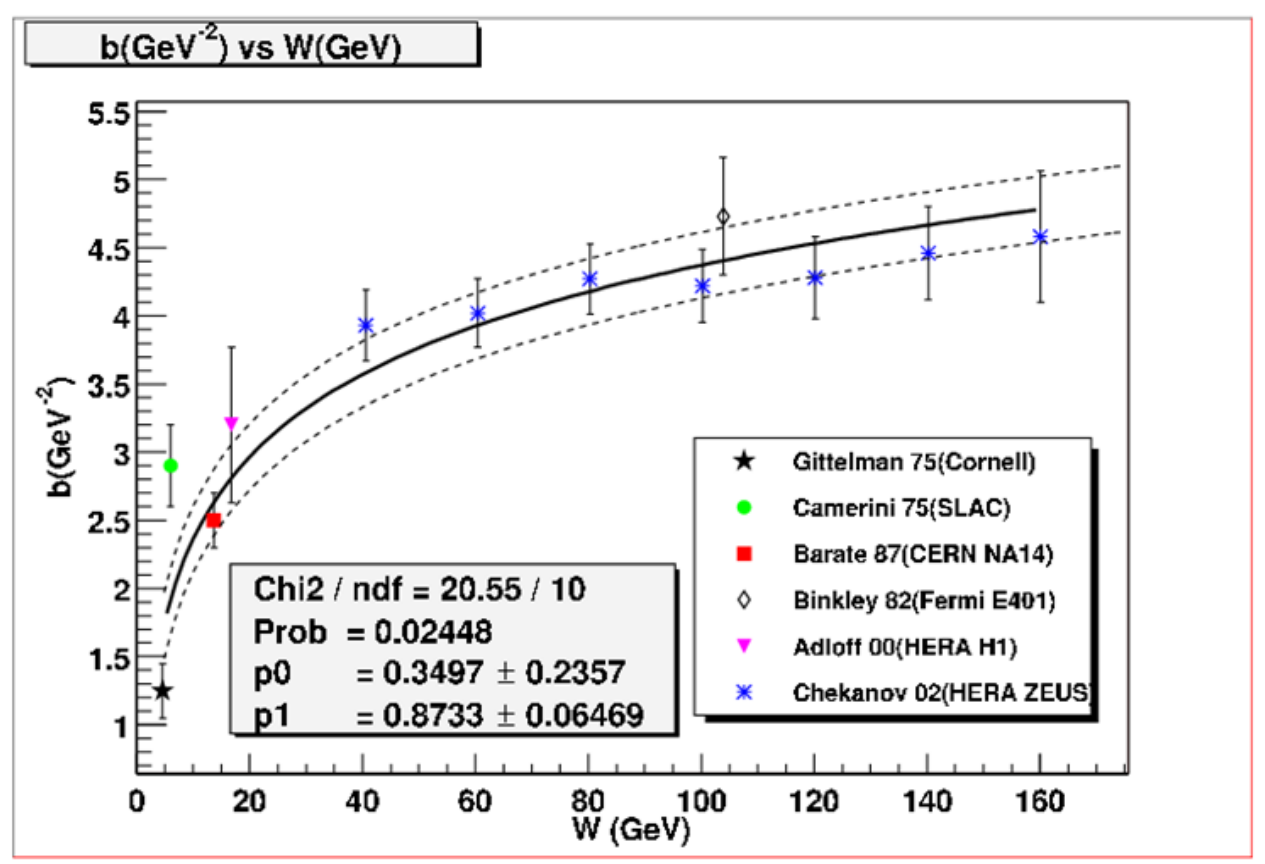

Figure 2: Slope parameter $b$ extracted from fits of the form $A e^{b t}$ to various $J / \psi$ photoproduction experiments, as a function of $W=\sqrt{s}$.

The total cross sections are shown in Fig. 3. The total cross sections drop very rapidly towards threshold, but then both the Cornell and SLAC single arm data (not shown) appear to flatten out and become almost independent of energy below $12 \mathrm{GeV}$. The two curves are from [8], corresponding to all of the cross section at $12 \mathrm{GeV}$ being described by 2-gluon or higher twist 3-gluon exchange, assuming that the effective 2-gluon and 3-gluon form factors are both given by $F^{2}=e^{1.13 t}$. A transition from the 2-gluon exchange process to the higher twist 3-gluon process near threshold provides one possible explanation of the flattening of the cross section near threshold. The 3-gluon process has a much weaker $s$ dependence because the momentum transfer is shared among more gluons, thus reducing the number spectators. This enhances the cross section by a factor of $(1-x)^{-2}$ where, in the definition from [8], the momentum fraction $x=\left(s_{t h}-m^{2}\right) /\left(s-m^{2}\right)$ is close to unity for threshold photoproduction. As shown in [12], the almost flat cross section near threshold can also be explained by 2-gluon exchange because, in their formalism, $x$ has a maximum value of about 0.8 , so factors such as $(1-x)^{2}$ do not cause a large threshold suppression. 


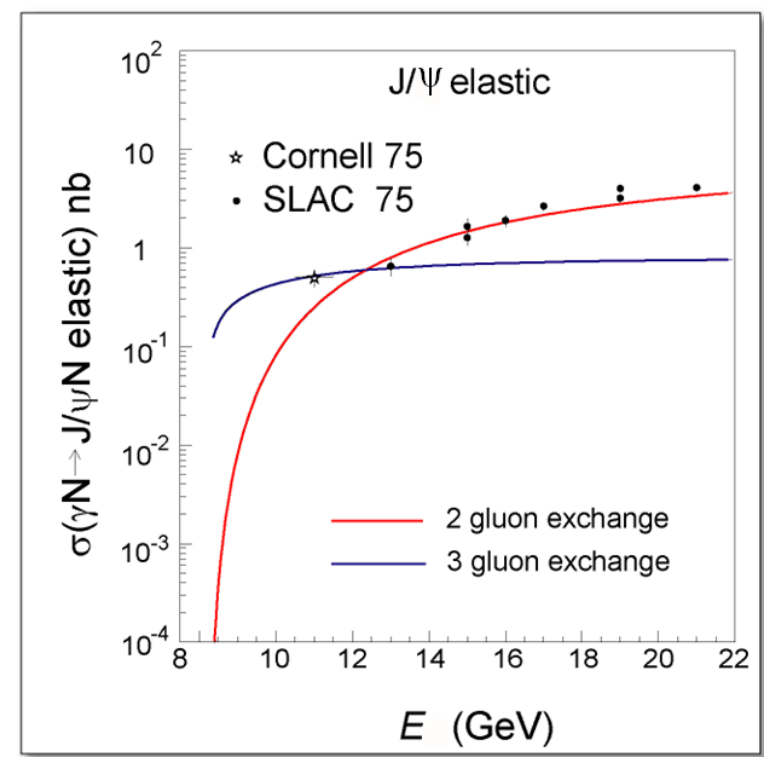

Figure 3: Total cross section for exclusive $J / \psi$ photoproduction from a proton as a function of photon energy. The steeper (flatter) curve is the simple 2-gluon (3-gluon) model of [8], normalized to the data at $12 \mathrm{GeV}$.

\subsubsection{E03-008}

Our exploratory $J / \psi$ measurement ran during the end of November and into December of 2004 in Jefferson Lab's Hall C. The SOS spectrometer was set up to measure negative leptons from the decay of $J / \psi$ meson, while the HMS spectrometer was set to measure the positive leptons from the decay. Since the SOS has a maximum central momentum setting of 1.7 $\mathrm{GeV}$, the angle had to be set to $53^{\circ}$. The HMS was parked at $24^{\circ}$ and the central momentum was set to $3.5 \mathrm{GeV} / \mathrm{c}$. Both spectrometers had standard triggers comprised of a coincidence of three out of four scintillator planes. To double our detection efficiency of the leptonic decays of the $J / \psi$, we needed to augment the detector package in the SOS spectrometer in Hall C. To reach the proper index of refraction for muon identification $(P \approx 1.7 \mathrm{GeV} / \mathrm{c})$, we needed about 1.7 atm of $\mathrm{C}_{4} \mathrm{~F}_{10}$. The original SOS cerenkov was designed to run at or below atmospheric pressure, hence we needed a new tank. We were able to have the cerenkov tank from the SLAC End Station A 1.6 GeV spectrometer shipped to Jefferson Lab. Over the summer of 2004, the PI and an MSU undergraduate student traveled to Jefferson Lab and worked on the design modification of the SLAC cerenkov. We developed a Monte Carlo to determine the position dependent lepton detection efficiency. We used this Monte Carlo to determine the necessary modifications to the tank windows. In addition to a Čerenkov signal, a minimum ionizing cut on each layer of the electromagnetic shower counter helped determine muon events. Hence both spectrometers had lepton particle ID detectors allowing coincident detection of lepton decays of vector mesons. The typical $J / \psi$ production run consisted of about $80 \mu \mathrm{amp}$ of electrons impinged on a $6 \%$ radiation length carbon target with a coincident trigger of the two spectrometers. We ran for four days in $J / \psi$ production mode and then spent a day for calibration runs. 


\section{Calibration Runs}

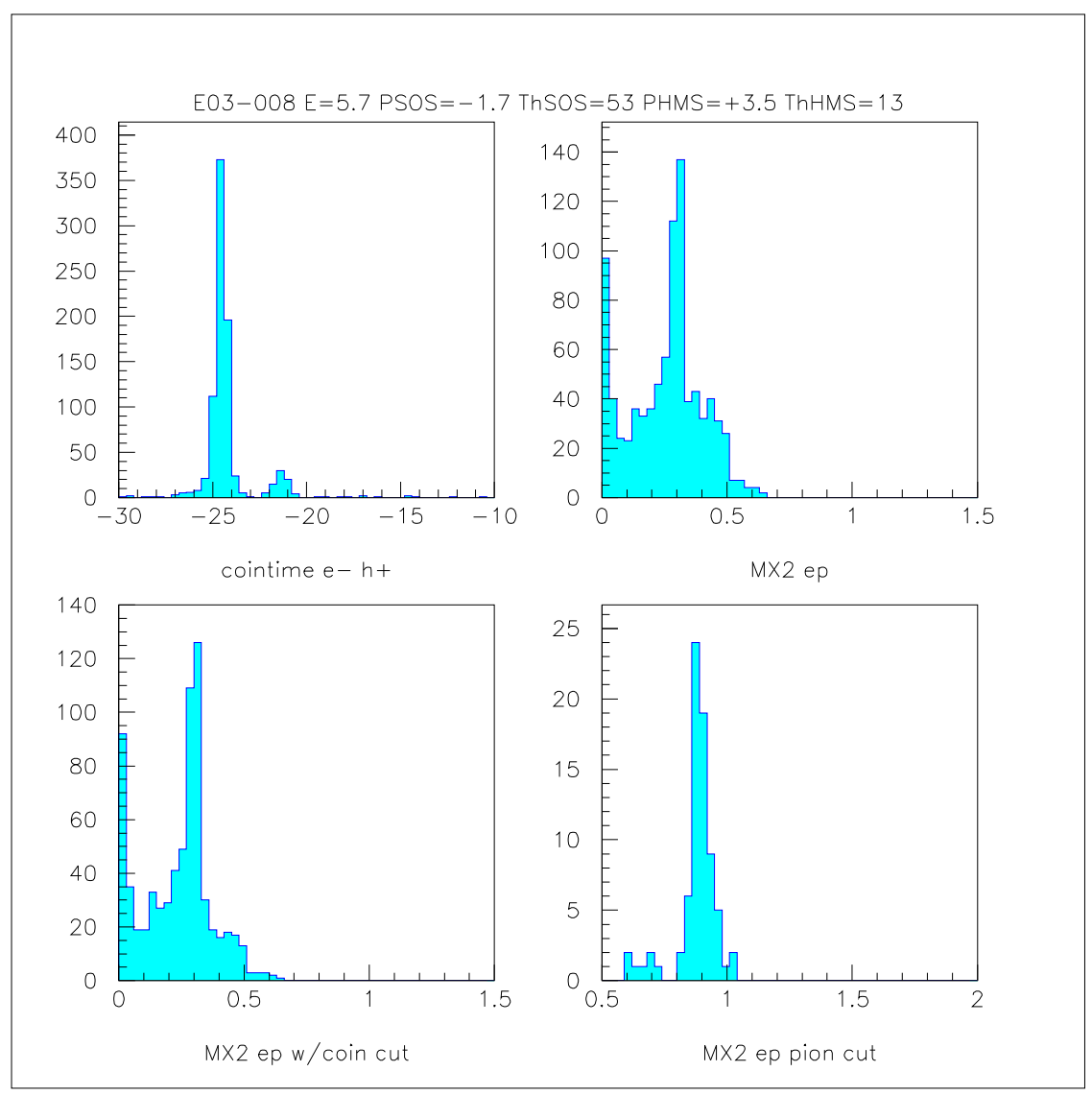

Figure 4: The proton and pion peaks are visible in the upper left coincidence time plot. The top-right plot is the square of the missing mass with no coincidence time cut and assuming the hadron has the mass of a proton. The bottom-left plot is the same missing mass but with a cut on the proton coincidence time. The bottom-right plot is the square of the missing mass assuming the hadron is a pion and with a cut on the pion peak in the coincidence time.

Before changing to other vector meson kinematics, we put in the liquid hydrogen target to check the spectrometer settings and resolutions by looking at electron-hadron coincidences. For the $J / \psi$ running the SOS spectrometer was setup to measure electrons, but now we were looking for hadrons ( $\pi^{+}$and protons) in the HMS. The spectrometer triggers remained the same, but differing particle ID cuts were used on the HMS data. The coincidence data from these runs are shown in Figure 4. The top-left plot shows the coincidence time for the electron-proton and the electron-pion, separated by about $2.5 \mathrm{~ns}$ as expected from the time-of-flight difference in the HMS at $3.5 \mathrm{GeV}$. The bottom-left plot is the missing mass squared assuming the hadron is a proton and a cut on the proton peak in the coincidence time spectrum. The $\pi^{0}$ and $\eta$ peaks are clearly visible and in the correct place. The bottom-right plot is the missing mass squared assuming the hadron is a pion and a cut on the pion peak in the coincidence time. The peak is at 0.9 , which is the neutron mass squared as expected. Hence, these calibration runs indicated the spectrometer settings were well understood. 
In addition to these runs, we adjusted the spectrometer momenta and angles to search for lepton pair decays of $\phi$ and $\omega$ mesons. By comparing these data to the Monte Carlo used for $J / \psi$ production, we could determine whether or not our technique for resolving the cross section was reasonable. Unfortunately, we did not run long enough to see a peak in the $\phi$ data, but there was a clear peak in the $\omega$ data as shown in Figure 5. For both the $\phi$ and $\omega$ production, the reaction was above threshold in a region where the cross sections are well known. The Monte Carlo predicted about $160 \omega$ events for the luminosity of the omega runs compared to about 145 detected events. This agreement between the $\omega$ data and Monte Carlo validated our technique for leptonic decays of vector mesons and gave us confidence that our $J / \psi$ predictions were reasonable.

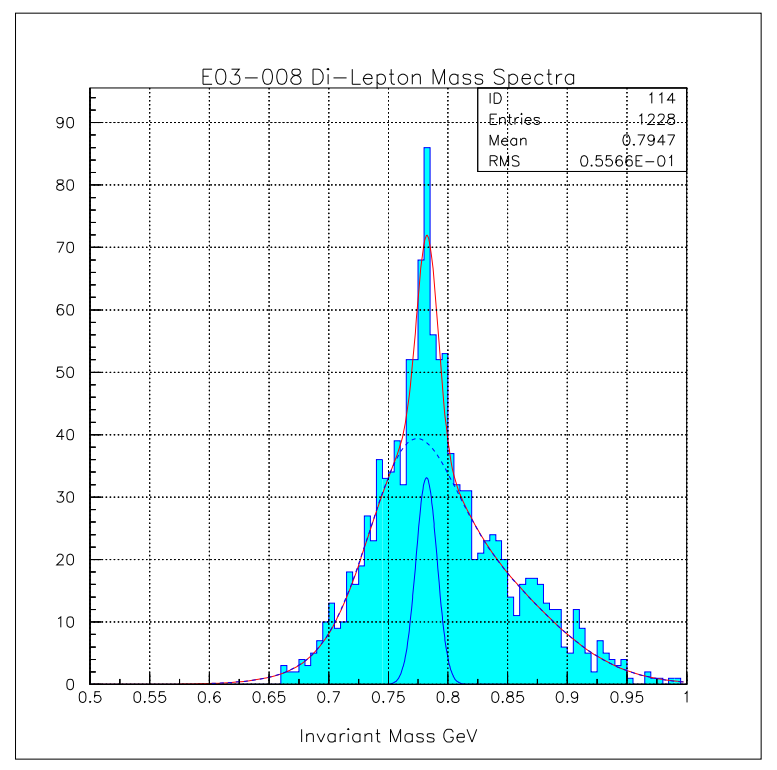

Figure 5: Plot of the invariant mass of the $e^{+} / e^{-}$coincident events from $\omega$ kinematics. There is a clear peak visible at the mass of the $\omega$ with the correct width $(\sim 8 \mathrm{MeV})$. The smooth background is roughly the shape of our acceptance with additional $\rho$ mesons. There are about 145 omega events in this spectrum.

\section{$J / \psi$ Data Analysis}

All the production runs were analyzed and the coincidence time spectra are shown in Figure 6. The rates were low enough that even the spectrum without any particle ID cuts had a very small accidental background. Electron particle ID cuts consisted of Cernekov signals and large energy deposited in the four layer electromagnetic shower counter from each spectrometer and a coincidence time cut. Muon particle ID cuts consisted of Cernekov signals and minimum ionization energy in each layer of the shower counters and a coincidence time cut. A double peak is seen in the coincidence spectrum when electron particle identification cuts are applied to the SOS arm(top left plot). The two peaks in the electron-hadron spectrum are due to the time-of-flight difference between the protons and pions. When electron particle ID cuts are applied to the HMS, the $e^{+} / e^{-}$spectrum has only one event, which is close to the true coincidence time. The $\mu^{+} / \mu^{-}$spectrum also has one event in coincidence time window. The reconstructed invariant mass of the lepton pairs is shown in Figure 6. The one electron 
pair event is at a mass of about $2.7 \mathrm{GeV} / c^{2}$, which is well below the $J / \psi$ mass and the muon spectrum matches the phase space spectrum shown in the top plots. These muon data probably came from pions which had decayed to muons in flight. The lone event in the $e^{+} / e^{-}$spectrum is either a Bethe-Heitler event or a $\pi^{+} / e^{-}$coincidence in which the pion was misidentified as an electron.
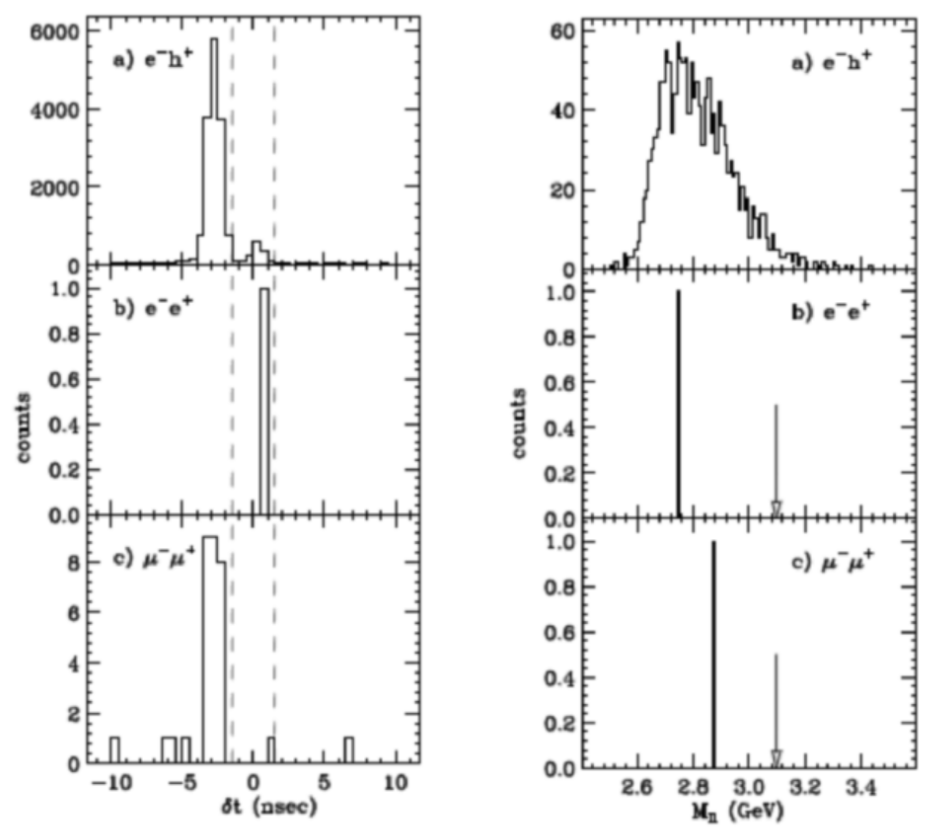

Figure 6: The plots on the left side are the coincidence time spectra for $J / \psi$ production runs. The plots on the right side are the reconstructed mass of the coincident particles from the two spectrometers for $J / \psi$ production runs. The top plots, a) " $e^{-} h^{+}$" have electron cuts on the SOS arm and no cuts on the HMS arm, while b) " $e^{+} e^{-}$" have electron cuts on both SOS and HMS arms, and c) " $\mu^{+} \mu^{-}$" have muon cuts in both spectrometers.

\section{$J / \psi$ Cross Section Analysis}

As a first pass to determine whether or not 2-gluon or 3-gluon production mechanisms dominate the production near threshold, we used our Monte Carlo to predict the rates of $J / \psi$ mesons observed from the sum of $e^{+} / e^{-}$and $\mu^{+} / \mu^{-}$final states for the conditions of our run for three cross section models. The models were:

$$
\begin{aligned}
\text { Model I: } \frac{d \sigma}{d t} & =a e^{b t} \\
\text { Model II: } \frac{d \sigma}{d t} & =\frac{a}{(1-b t)^{4}} \\
\text { Model III: } \quad \frac{d \sigma}{d t} & =\frac{a(1-x)^{2}}{(1-b t)^{4}}
\end{aligned}
$$

The motivation for the above models is that, near threshold, charm probes the $x \simeq 1$ target 
configuration, where

$$
x=\frac{\left(m_{p}+m_{J / \psi}\right)^{2}-m_{p}^{2}}{s-m_{p}^{2}} .
$$

Hence the spectator partons carry $x \rightarrow 0$ fraction of the target momentum. Therefore, there is a suppression factor as $x \rightarrow 1$ of $(1-x)^{2 n_{s}}$ where $n_{s}$ is the number of spectator partons. Models I and II correspond to 3-gluon exchange with an exponential and dipole form factor, respectively and model III corresponds to a 2-gluon exchange with a dipole form factor. In addition to the above models, the off-shell effects of the struck nucleon (with momentum $\left.P_{m}\right)$ were treated in several ways. Since there is insufficient data from $\mathrm{C}\left(\mathrm{e}, \mathrm{e}^{\prime}, \mathrm{p}\right) \mathrm{X}$ to constrain the spectral function beyond $P_{m}=0.6 \mathrm{GeV}$, we used two "eyeball" extrapolation. These extrapolations are similar to the nuclear matter extrapolations in Benhar [13]. We used two different prescriptions for the missing energy distribution at high missing momentum, one was to "freeze" the $E_{m}$ distribution to that of Benhar et al. [14] at $P_{m}=0.8 \mathrm{GeV}$ (the highest provided in their fit). The other prescription was to "shift" the $E_{m}$ distribution by

$\sqrt{m_{p}^{2}-P_{m}^{2}}-\sqrt{m_{p}^{2}-0.8^{2}}$, which corresponds to the "ridge" observed at lower missing energies. For the three different models and four combinations of treating the high momentum tail and the missing energy of the struck nucleon, we used the Monte Carlo to determined the parameter $a$ for each $b$ ranging below $3.0\left(\mathrm{GeV}^{-2}\right)$ such that we would get a cross section of $0.7 \mathrm{nb}$ (the Cornell value). Then we determined how many counts we would have observed for our integrated luminosity. This is shown in Figure 8.

In summary, E03-008 was an exploratory measurement that was sensitive to short range effects in the nuclei. The results are consistent with predictions of quasi-free $J / \psi$ production using a variety of elementary free nucleon cross section models and several educated guesses for the high missing momentum and energy distributions in carbon. Upper limits can be set on exotic mechanisms with appropriate assumptions for cross section and spectral function (e.g., hidden color, 3 gluon exchange, intrinsic charm). The interpretations of this experiment will certainly benefit from near-threshold measurements of $J / \psi$ production once the Jefferson Lab beam energy increases above threshold.

\subsection{Photoproduction of $J / \psi$ near threshold}

The threshold for photproduction of $J / \psi$ meson on a free proton is about $8.2 \mathrm{Gev}$. With the high energy upgrade at the Jefferson Lab providing up to $11 \mathrm{GeV}$ electrons, a vast array of charm measurements are possible. In the following discussion, one such measurement will be detailed. Threshold charm production on a nucleus can give information on the $J / \psi-\mathrm{N}$ interaction, which is important in understanding the actual strength of the QCD van der Waals potential due to gluon exchange between the meson and nucleon [15]. Additionally, knowledge of the propagation of the $J / \psi$ 's in nuclei is relevant to the interpretation of relativistic heavy ion collisions, since it is believed that $J / \psi$ suppression may be a signal for the formation of quark-gluon plasma. The standard method to extract this quantity has been to measure the nuclear dependence of $J / \psi$ production. The majority of these $A$-dependent $J / \psi$ production experiments have been measured at high energy, while the only near-threshold experiment was performed using $20 \mathrm{GeV}$ photons. This $20 \mathrm{GeV}$ SLAC experiment measured 

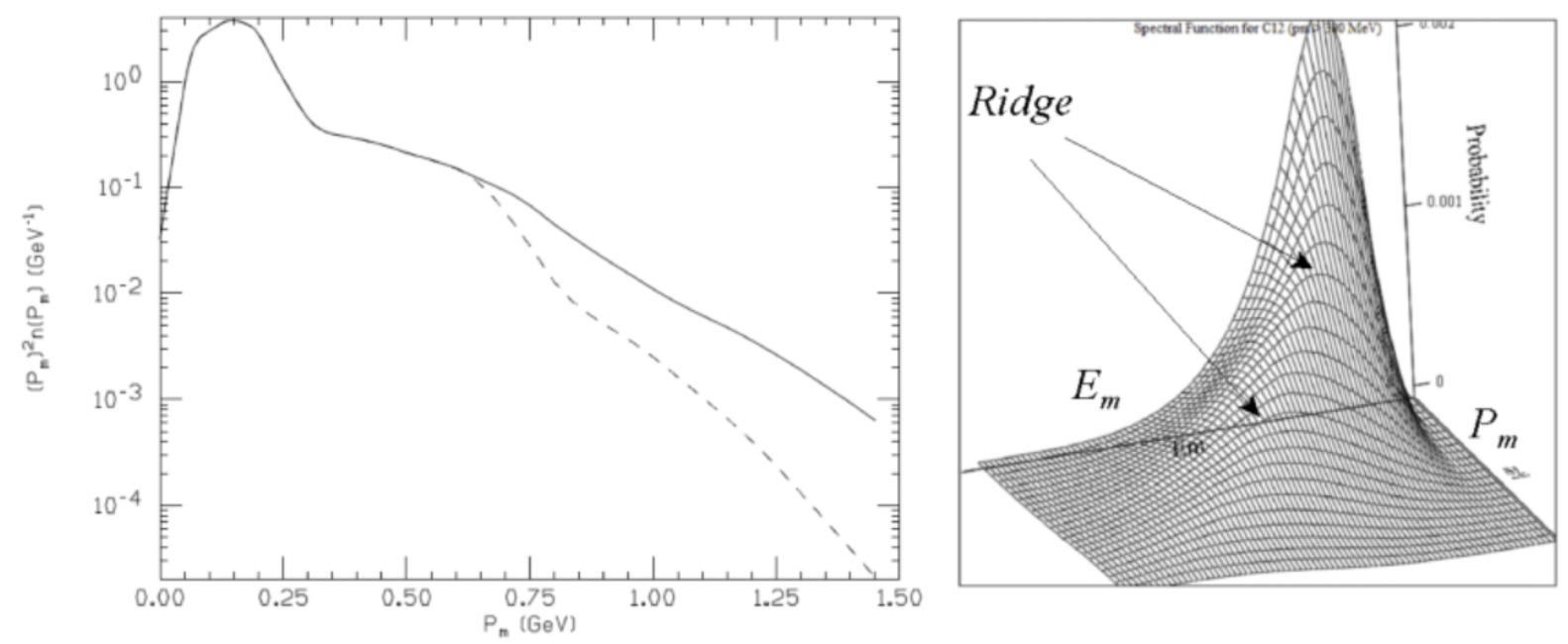

Figure 7: The figure to the left is the integrated missing energy $\left(E_{m}\right)$ probability of finding a nucleon with missing momentum $P_{m}$ as a function of $P_{m}$. The solid line is the "high" extrapolation and the dashed line is the "low" extrapolation. The figure to the right is the spectral function for carbon from O.Benhar et al.

$\sigma_{J / \psi N}=3.6 \pm 0.8 \pm 0.5 \mathrm{mb}[16]$; whereas theory predicts this cross section to be higher, about $7 \mathrm{mb}$ [17]. It is unclear whether the SLAC determination of $\sigma_{J / \psi}$ corresponds to the physical $\sigma_{J / \psi}$, due to the fact that at these energies the $J / \psi$ may still be formed outside the nucleus. In addition, due to the low rate, they only observed one muon from from the $J / \psi$ leptonic decay, thus had very large background subtractions. Hence, a new measurement of the nuclear dependence of threshold $J / \psi$ photoproduction could resolve this issue.

The production mechanism of $J / \psi$ mesons from nuclei can be split up into three processes: coherent elastic, quasi-elastic, and inelastic scattering. In coherent production, the photon fluctuates to an off-shell $c \bar{c}$ pair and then scatters elastically from the nucleus. At threshold, $t_{\min }$ is about $1 \mathrm{GeV}$, hence, coherent production can be neglected and since there is little extra energy at threshold, the dominating process is quasi-elastic production. The leadingorder elastic contribution is two gluon exchange [17]. Inelastic scattering is believed to be described by the photon-gluon fusion mechanism [18], where the photon interacts with the gluon content of the nucleon through the sub-process, $\gamma g \longrightarrow c \bar{c}$.

Following the convention of Brodsky and Mueller [19], the interaction is split into two time scales, production time $\left(\tau_{P}\right)$ and formation time $\left(\tau_{F}\right)$. In the target rest frame, the production time is the time of the hard interaction, while the formation time is the time that the produced partonic system takes to reach the physical configuration of the hadron. The production time or, as it is sometimes called, the coherence time, has the following form in the target rest frame:

$$
\tau_{P} \simeq \frac{2 \nu}{4 m_{c}^{2}}+Q^{2} \stackrel{Q^{2}=0}{\longrightarrow} 0.04 \nu \text { fm } \mathrm{GeV}^{-1} \quad \text { where } \nu \text { is the photon energy }
$$



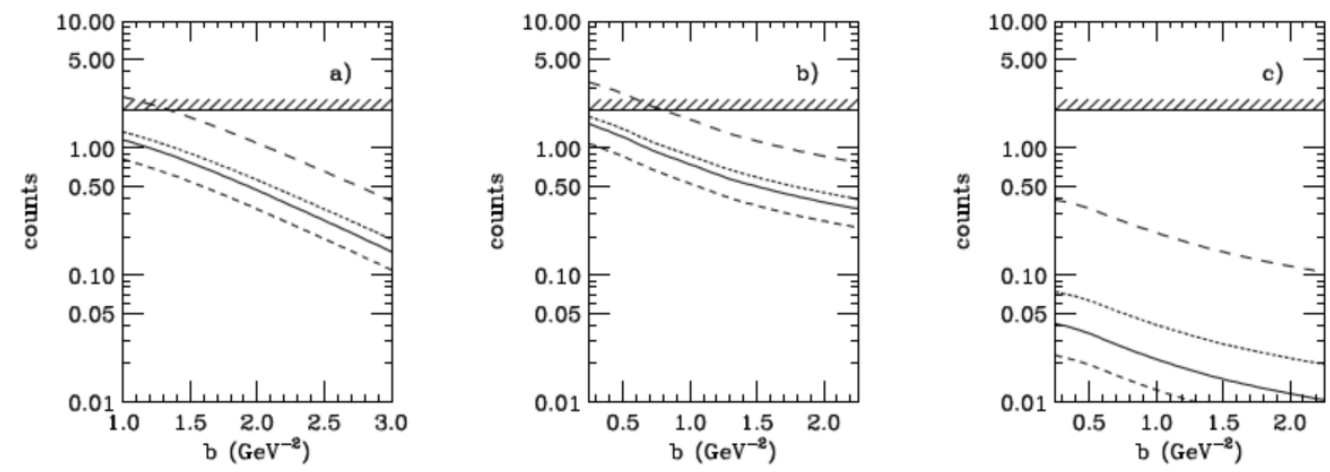

Figure 8: Upper Limit Cross Section for the three models. The solid line is the "high" extrapolation with Missing Energy $\left(E_{m}\right)$ shift, the dashed line is the "high" extrapolation without the $E_{m}$ shift, the short dashed line is the "low" extrapolation with $E_{m}$ shift, and the dotted line is the "low" extrapolation without the $E_{m}$ shift.

This is the lifetime of the hadronic fluctuation and for energies below $150 \mathrm{GeV}$ the interaction involves a single nucleon in the nucleus. The formation time according to Kopeliovich and Zakharov [20] has the form:

$$
\tau_{F} \simeq \frac{2}{m_{\psi^{\prime}}-m_{J / \psi}}\left(\frac{\nu}{2 m_{c}}\right) \simeq 0.2 \nu \mathrm{fm} \mathrm{GeV}^{-1}
$$

To probe the $J / \psi$-nucleon cross section, the formation time must be less than the size of the nucleus. The Vector Meson Dominance/Glauber prediction shown below is valid for $\tau_{F} \ll R_{A}$ where $R_{A}$ is the radius of the nucleus. The transparency factor is given by

$$
T_{N}(A) \simeq 1-\frac{1}{2 A} \sigma_{J / \psi N} \int d^{2} b T(b)^{2}
$$

where $T(b)$ and $b$ are the optical thickness of the nuclei and the impact parameter. Thus on can measure the cross section per nucleon on two nuclear targets and form a ration to extract the $J / \psi$-N cross section, $\frac{\sigma_{A_{1}} / A_{1}}{\sigma_{A_{2}} / A_{2}}=\frac{T_{N}\left(A_{1}\right)}{T_{N}\left(A_{2}\right)}$. Forming this ratio enables the extraction of the $\sigma_{J / \psi N}$ without measuring absolute cross sections. Since there is a large uncertainty in the formation time, it is advantageous to measure $J / \psi$ production with a low energy probe, hence maximizing the chance that the $c \bar{c}$ forms into a $J / \psi$ within the nucleus. As an example, using the formation time from above, the $J / \psi$ 's produced in the SLAC near-threshold experiment would have a formation time of $4 \mathrm{fm}$. While this is consistent with the typical nuclear size, it is unclear whether or not the application of the Glauber model is valid.

\subsubsection{Proposed Measurement}

Using the high luminosity $11 \mathrm{GeV}$ Jefferson Lab beam, and the pair of magnetic spectrometers in Hall-C, one can investigate the $A$-dependence of quasi-free $J / \psi$ photoproduction with an average photon energy near $11 \mathrm{GeV}$. The PI is a co-spokesperson on an experiment (E12-07-106 [21]) to measure $J / \psi$ photoproduction near threshold and it was conditionally 
approved by the Program Advisory Committee. Analysis of these data will permit a greatly improved determination of the fundamental $J / \psi$ total cross section. In order to reduce modeling uncertainties, we will also make precision measurements of the $(s, t)$ dependence of the elementary $J / \psi$ photoproduction cross section on the proton, improving the existing data and extending them to the threshold region. Comparisons to models will allow improved determination of the reaction mechanism. The principal requirements beyond the planned standard Hall C configuration are for a set of nuclear targets and an external radiator for the liquid hydrogen target.

\subsection{Qweak Target Work}

The MSU group is one of the lead groups responsible for the design, construction, and commissioning of the world's highest power liquid hydrogen cryotargets. The following subsections give a brief outline of the goals of the $Q_{w}$ expermiment and then follows up with the target progress made over the last six months.

\subsubsection{Background to Qweak Measurement}

\section{Introduction}

Figure 9 shows the Standard Model prediction in a particular scheme for $\sin ^{2} \theta_{W}$ together with existing and proposed world data. The precise data at the $Z^{0}$ pole sets the overall magnitude of the curve, but to test the shape, one needs precise measurements at differing $Q^{2}$. The Standard Model evolution predicts a shift of $\Delta \sin ^{2} \theta_{W}=+0.007$ at low $Q^{2}$ with respect to the value of $0.23113 \pm 0.00015$ at the $Z_{0}$ pole. Measurements at low $Q^{2}$ that differ from the evolved curve would indicate new physics, while measurements that agree with the evolved curve would place constraints on possible Standard Model extensions. Currently, three measurements off the $Z^{0}$ pole exist which test the running of the weak angle, atomic parity violation (APV), polarized Møller scattering (E-158), and neutrino-nucleus deep inelastic scattering $(\mathrm{NuTeV})$. Apparently, the theoretical uncertainties in the APV determination are not completely under control, but if one uses the most precise value for the polarizability $[22,23]$ with the most current theory calculation [24], the determination of $\sin ^{2} \theta_{W}$ is about $1.6 \sigma$ below the SM prediction. The determination of the SLAC polarized Møller scattering experiment E-158 is in agreement with the predicted value [26]. The determination of $\sin ^{2} \theta_{W}$ from the $\mathrm{NuTeV}$ Collaboration is about $3 \sigma$ above the predicted value, which may be indicating new physics [25]. There is an approved complimentary experiment: the Jefferson Lab $Q_{W}^{p}$ [27] measurement. The proposed measurement of $Q_{W}^{p}$ will have significantly smaller statistical and systematic errors and the theoretical interpretation is more straightforward than in the APV experiments.

\section{Physics Background}

The asymmetry $A_{L R}\left({ }^{1} H\right)$ is the ratio of the difference over the sum of elastically scattered longitudinally polarized electrons with positive and negative helicity from unpolarized pro- 


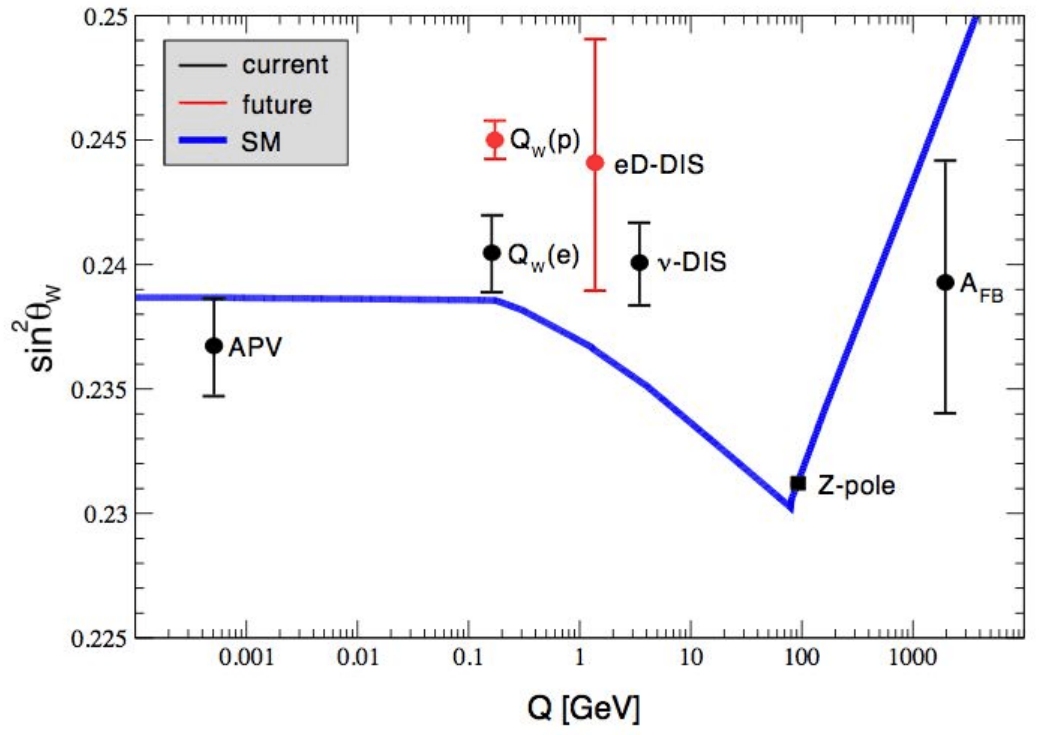

Figure 9: Calculated running of the weak mixing angle in the Standard Model, defined in the modified minimal subtraction scheme. The black data points show the current situation, while the red (gray) data points (with arbitrarily chosen vertical locations) refer to the asymmetry measurements proposed at the Jefferson Lab and deep inelastic e-D scattering. The previous measurements are determinations from atomic parity violation (APV), Møller scattering (E-158), deep inelastic neutrino-nucleus $(\mathrm{NuTeV})$, and from $Z^{0}$ pole asymmetries $(\mathrm{LEP}+\mathrm{SLC})$ (from ref. [27]).

tons:

$$
A_{L R}=\frac{\sigma_{+}-\sigma_{-}}{\sigma_{+}+\sigma_{-}}
$$

The asymmetry, expressed in terms of Sachs electromagnetic form factors $G_{E}^{\gamma}, G_{M}^{\gamma}$, the weak neutral form factors $G_{E}^{Z}, G_{M}^{Z}$, and the neutral weak axial form factor $G_{A}$, has the following form $[28]$

$$
A_{L R}=\left[\frac{-G_{F} Q^{2}}{4 \pi \alpha \sqrt{2}}\right]\left[\frac{\epsilon G_{E}^{\gamma} G_{E}^{Z}+\tau G_{M}^{\gamma} G_{M}^{Z}-\left(1-4 \sin ^{2} \theta_{W}\right) \epsilon^{\prime} G_{M}^{\gamma} G_{A}^{Z}}{\epsilon\left(G_{E}^{\gamma}\right)^{2}+\tau\left(G_{M}^{\gamma}\right)^{2}}\right]
$$

where

$$
\epsilon=\frac{1}{1+2(1+\tau) \tan ^{2}(\theta / 2)}, \quad \epsilon^{\prime}=\sqrt{\tau(1+\tau)\left(1-\epsilon^{2}\right)}
$$

are kinematic quantities, $Q^{2}$ is the four momentum transfer, $\tau=Q^{2} / 4 M^{2}$ where $M$ is the proton mass, and $\theta$ is the laboratory electron scattering angle. In the case of forward angle scattering where $\theta \rightarrow 0, \epsilon \rightarrow 1$, and $\tau \ll 1$, the asymmetry has the form [29]

$$
A_{L R}=\left[\frac{-G_{F}}{4 \pi \alpha \sqrt{2}}\right]\left[Q^{2} Q_{W}^{p}+F^{p}\left(Q^{2}, \theta\right)\right] \longrightarrow\left[\frac{-G_{F}}{4 \pi \alpha \sqrt{2}}\right]\left[Q^{2} Q_{W}^{p}+Q^{4} B\left(Q^{2}\right)\right]
$$


where $F^{p}\left(Q^{2}\right)$ is a form factor and $B\left(Q^{2}\right)$ is the leading term from nucleon structure defined in terms of neutron and proton electromagnetic and weak form factors. If one neglects radiative corrections, then the leading term is $Q_{W}^{p}=1-4 \sin ^{2} \theta_{W} . B\left(Q^{2}\right)$ can be determined from extrapolations from the ongoing PV program at higher $Q^{2}$, and given the expected precision of these experiments the optimal $Q^{2}$ is about $0.03(\mathrm{GeV} / \mathrm{c})^{2}$.

\section{Jefferson Lab $Q_{W}^{p}$}

The layout of the experiment is shown in Figure 10. It consists of a toroidal magnet which will focus elastically scattered electrons onto a set of 8 rectangular quartz Čerenkov detectors. The acceptance averaged asymmetry in this design is $-0.28 \mathrm{ppm}$. The asymmetry will be measured with $\pm 2 \%$ statistical and $\pm 2 \%$ systematic errors in a 2200 hour run with $180 \mu \mathrm{A}$ of $80 \%$ polarized electrons on a $35 \mathrm{~cm}$ liquid hydrogen $\left(\mathrm{LH}_{2}\right)$ target . This measurement will determine the proton's weak charge with $\simeq 4 \%$ combined statistical and systematic errors, which corresponds to an error on $\sin ^{2} \theta_{W}$ of $\pm 0.3 \%$ at low energy.

\section{Major Experiment Component Status}

Target(Design)

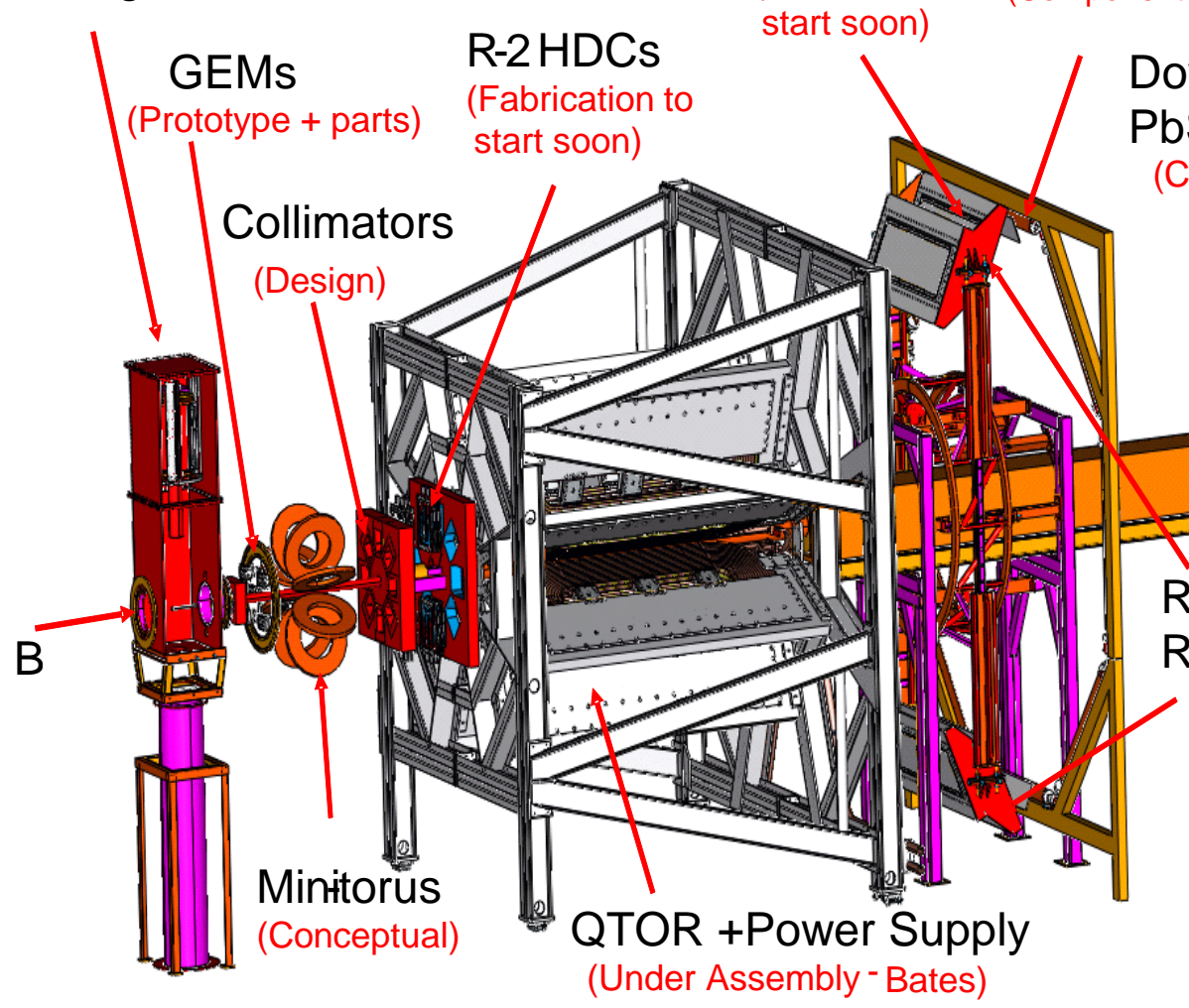

R-3 VDC Main Detectors (Fabrication to (Components at JLab)

Downstream PbShielding (Complete)

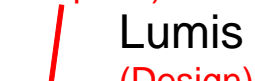
(Design)

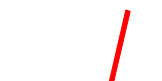

Scanner

(Design)

Figure 10: 3-D CAD drawing of experimental setup showing the target, collimation, shielding, electron trajectories, and detectors. 


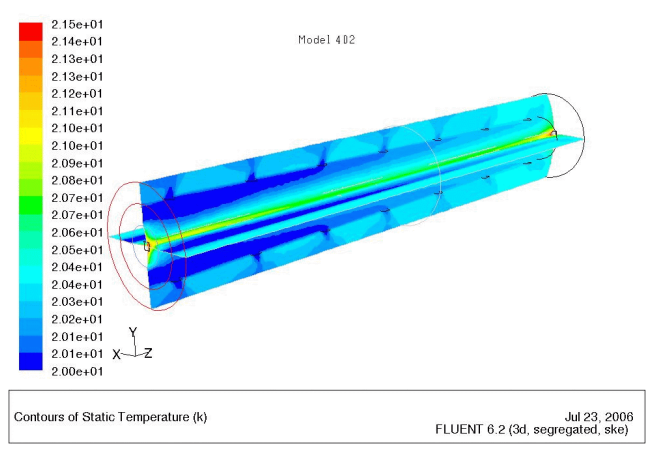

(a) G0-type cell

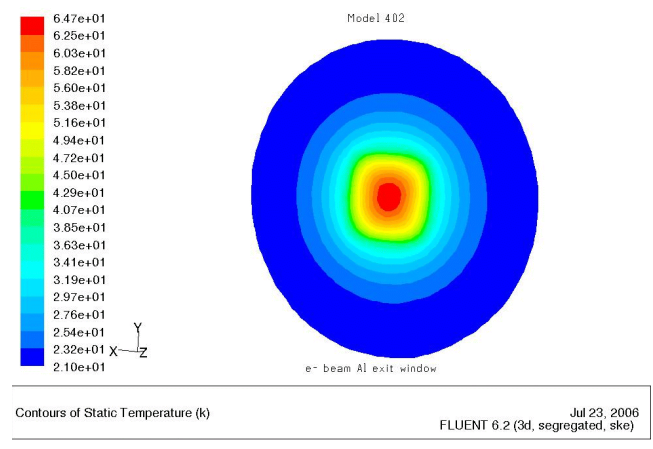

(b) Exit window

Figure 11: Temperature profile along cell and at window

\subsubsection{Introduction to MSU Target Work}

The MSU group has been involved in numerous target projects involving the cell design and simulations, heat exchanger calculations, design and heat transfer calculations for the high power heater, designing the motion mechanism and designing the solid targets needed for the experiment. The following subsections detail much of that effort.

\subsubsection{Cell Design}

In the summer of 2006, preliminary results from fluid dynamics modeling, primarily from the University of New Hampshire group, were coming in. They looked at several variations of our default G0-like longitudinal cell design initially. All the designs indicated hot spots at the beam entrance and exit windows, as expected, but the entrance hot spots were particularly bad. As one could guess and the model confirmed, the flow is poor at the entrance window. This endemic problem with the basic longitudinal cell geometry and more realistic volumetric flow rates in the target loop ${ }^{\dagger}$ caused us to rethink the overall cell design.

At the risk of losing the cell's azumuthal symmetry, we started looking into purely transverse flow cell designs. Whereas in the longitudinal cell, the inlet and outlet manifolds are upstream of the cell, in the transverse cells the manifolds must be along the cell. The radii of the cells must be made larger to move this extra material out of the acceptance. GEANT studies, currently lead by the MSU group, have been done and are not showing any large effect in the rates seen at the detectors. Even at this basic level, the transverse flow designs are showing about a factor of two less bulk temperature rise in the volume of hydrogen intersecting the beam. In all cases the window temperatures exceed the boiling temperature of hydrogen, hence there will be some amount of film boiling near the windows. Since there is clearly a phase transition at the windows, the CFD calculations are probably the least accurate in that region, but greater understanding of the flow is a huge improvement in how cells are designed. We investigated new transverse designs to get more flow directed at the windows.

\footnotetext{
${ }^{\dagger}$ The original planned volumetric flow rate of the hydrogen was $25 \mathrm{l} / \mathrm{s}$, but the heat load from the viscous heating in the loop was too high. Hence, this rate will need to be reduced to $15 \mathrm{l} / \mathrm{s}$.
} 


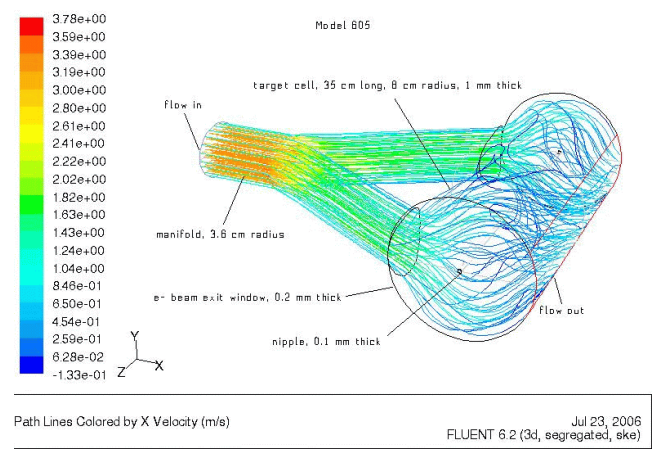

(a) Streamline velocity $(\mathrm{m} / \mathrm{s})$

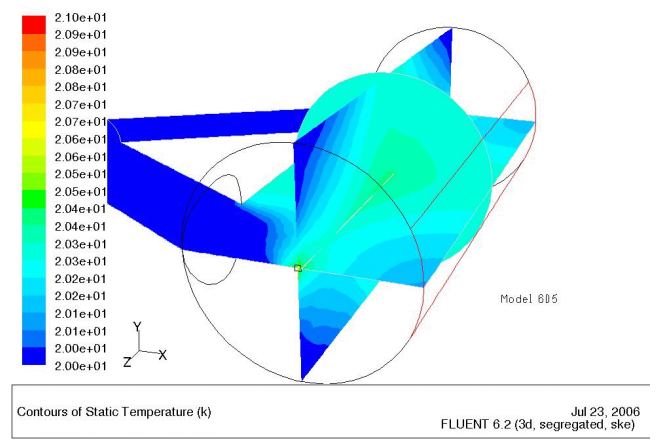

(b) Temperature profile

Figure 12: Streamline velocities and temperature profile along transverse cell.

The MSU group looked into more realistic cell designs with realistic downstream windows ${ }^{\dagger}$. The stress on the window is roughly proportional to the radius of curvature of the window and inversely proportional to the window thickness. Hence, one needs to balance the amount of curvature at the window, which will affect the flow at the window, with minimizing the window thickness, which affects the multiple scattering.

\subsubsection{High Power Heater}

The other major piece of work completed during this grant period was the high power heater to be used to control the target temperature and replace the roughly 2200 Watts of beam load heating when the beam trips. The MSU group did the original heat transfer calculations to determine the required characteristics of the heater. The target group at JLab designed the original heater that was to be located in one of the transition cone pieces of the heat exchanger. The MSU group built that heater in the previous reporting period (see Figure 13 (a)). Unfortunately, due to excess liquid hydrogen in the target, we had to eliminate one of the heat exchangers and fill most of the volume in these transition pieces. This required the heater to be located in a section of 3" pipe of the loop. The MSU group performed the necessary heat transfer calculations and designed the new heater to reside in a section of 3" pipe. This was a non-trivial task, since we had two major new constraints. Based on the characteristics of the original heater (i.e., its electrical resistance and the pressure drop of the hydrogen through the heater) the pump was designed for a maximum head and two DC power supplies were purchased to operate the original heater. Hence, we had to design a heater that was to operate in faster flowing hydrogen, needed to deliver $2500 \mathrm{~W}$ without boiling the hydrogen, with a resistance of $1.2 \Omega$, and have a pressure drop less than 0.3 psi. We accomplished this with a four layer heater using 13 AWG Nichrome wire (see Figure 13 (b)).

Substantial effort was put into this new heater, in fact, more than was necessary due to a misstep regarding computational fluid dynamics (CFD) simulations done at JLab. The heat transfer calculations done at MSU indicated that the heat flux at maximum power was

\footnotetext{
${ }^{\dagger}$ The current modeled transverse cells have flat windows. To achieve this they would need to be unacceptably thick.
} 
well below the critical heat flux for liquid hydrogen, hence we did not expect film boiling in the loop. But due to a mistake in how the heat transfer was treated in the Nichrome wire, the CFD simulations were showing hot spots where the wire went through the G10 holes. Therefore, we put a lot of effort into resolving that issue. We produced a second four layer heater with G10 of half the thickness of the first (1/16" instead of $1 / 8$ "), used larger holes, and cut slots between adjacent holes to promote liquid flow where the wire penetrated the G10 boards. In parallel, the Jlab group designed a two layer heater which seemed to perform better, of course, mainly because the heat transfer in the wires was now done properly. So we embarked on a plan to build this two layer model and test the two locally at MSU. Once the mistake was found and the four layer model was redone, it was shown to work well, without hot spots. Hence, MSU has produced both the production heater, which was shipped to JLab in January, 2009, and a spare (with thicker G10 and no slots between the holes).

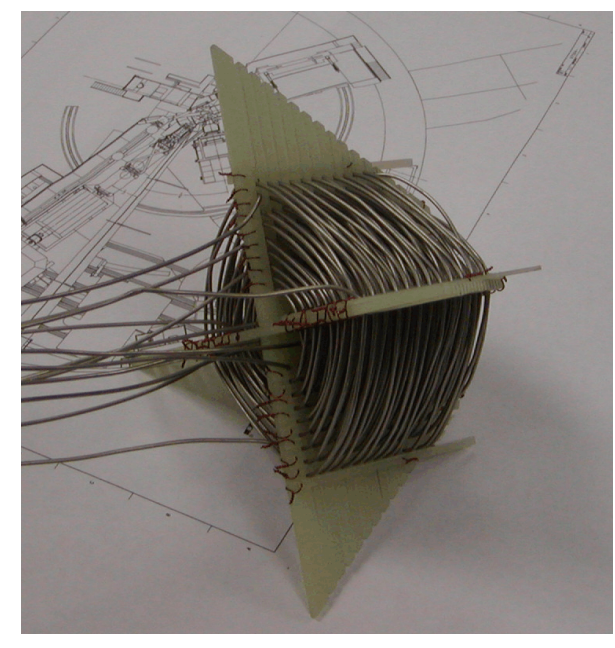

(a) Original Heater

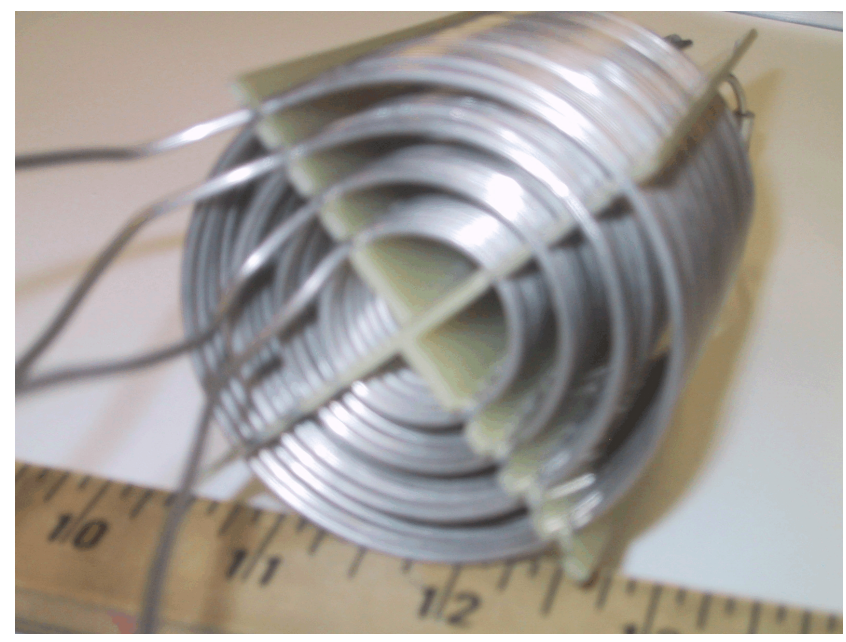

(b) New Heater

Figure 13: The original heater built by MSU to reside in transition piece of heat exchanger and the new heater to reside in 3 " loop pipe.

\subsubsection{Motion Mechanism}

One of the PI's first tasks for the $Q_{w}$ Collaboration was to design the motion mechanism for the cryogenic hydrogen target. The conceptual design was produced in late 2005 and 2006 and the following subsections describe that work. The final design was very similar with one major difference, it did not use three lead screw slides in the chamber, but rather had just two slides and an external linear actuator that provided the motion.

\section{Original Conceputal Motion Mechanism Design}

A top and side view of a rough drawing of the motion mechanism are shown in Figures 14 and 15. More accurate drawings exist in the Hall C CAD system, but the general ideas about how the system will work can be adequately described with these crude drawings. The vertical motion will be similar to the standard Hall $\mathrm{C}$ cryotarget, with three elevators/slides. 


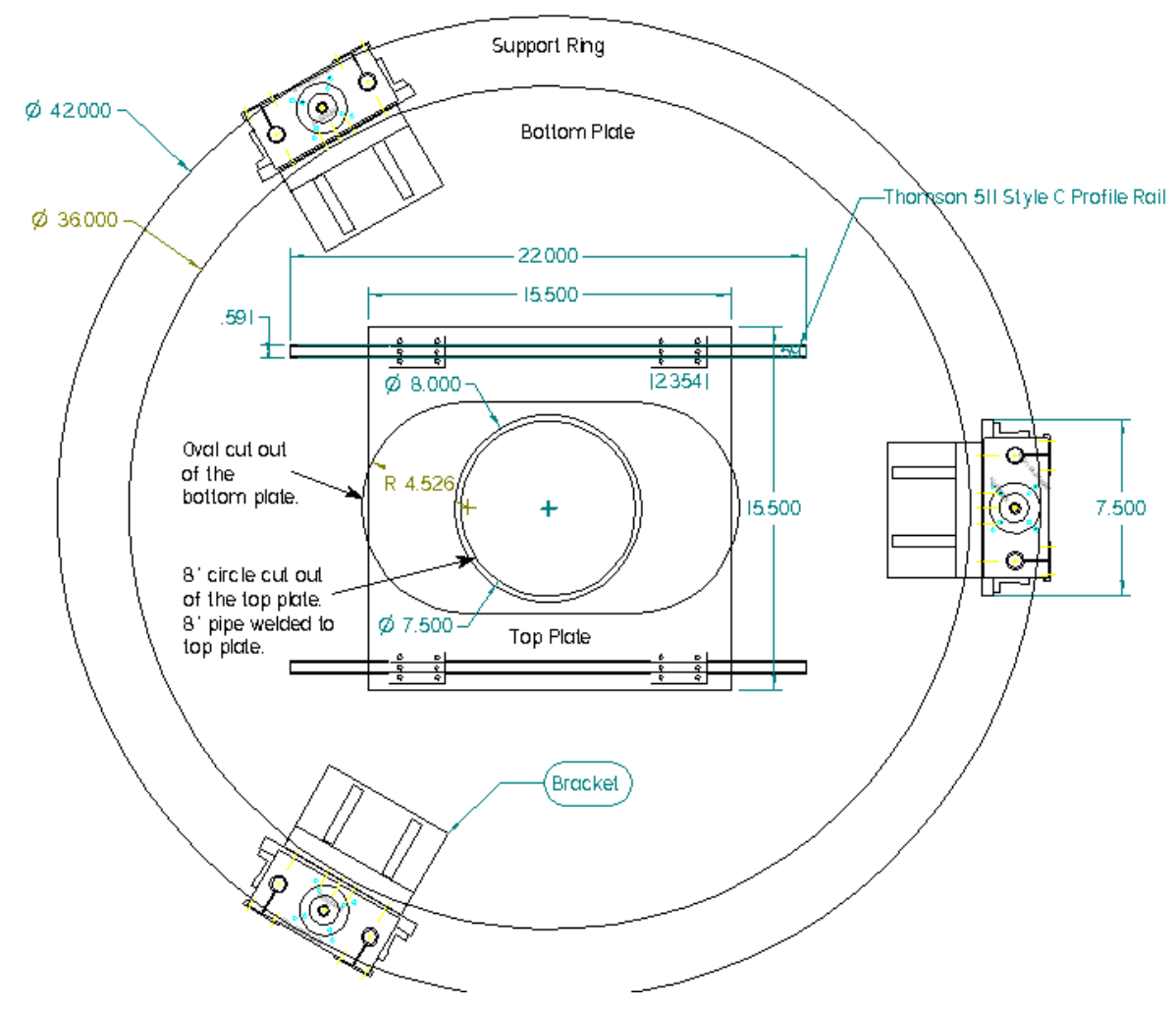

Figure 14: A top view of the rough drawing of the major elements of the motion mechanism.

The elevators will be rigidly held by a top and bottom support ring. In the CAD system, there are steel beams held by the support rings, and the elevators are attached to the beams. This may provide an easier way to align the elevators and also cost savings, since we can purchase shorter elevators. We are currently thinking of purchasing Thomson SuperSlide* 2RB continuously supported systems with integral ball screw assembly and T-slot carriage. The exact system will be determined once we have a more accurate idea of the mass on the carriage and the compressive load on the ball screw. They have standard sizes and lengths which will meet all of our possible needs. Since the elevators will be in vacuum, lubrication is an important consideration. Thomson does provide a low vapor pressure lubricant which will exceed our needs. Although, there may be less expensive solutions.

The ball screw shafts will couple to ferrofluid vacuum feed-throughs which penetrate the top of the vacuum chamber. These feed-throughs have a direct shaft and hence can provide high torque and a very good vacuum seal without the need for o-rings, as long as the magnetic fields around them are small. The top of the chamber will be sufficiently far from either the mini-torus or the main magnet, hence should operate without a problem. The shafts of the feed-throughs will couple to 50-1 gear boxes and will be coupled to one BDS5 motor via a sprocket system similar to the existing system in Hall C. In addition, each elevator will have a brake and possibly an encoder.

"L" brackets will be attached to the elevator slides and these brackets will support a circular stainless steel plate (called the bottom plate in the figures). This plate will have an oval cut 


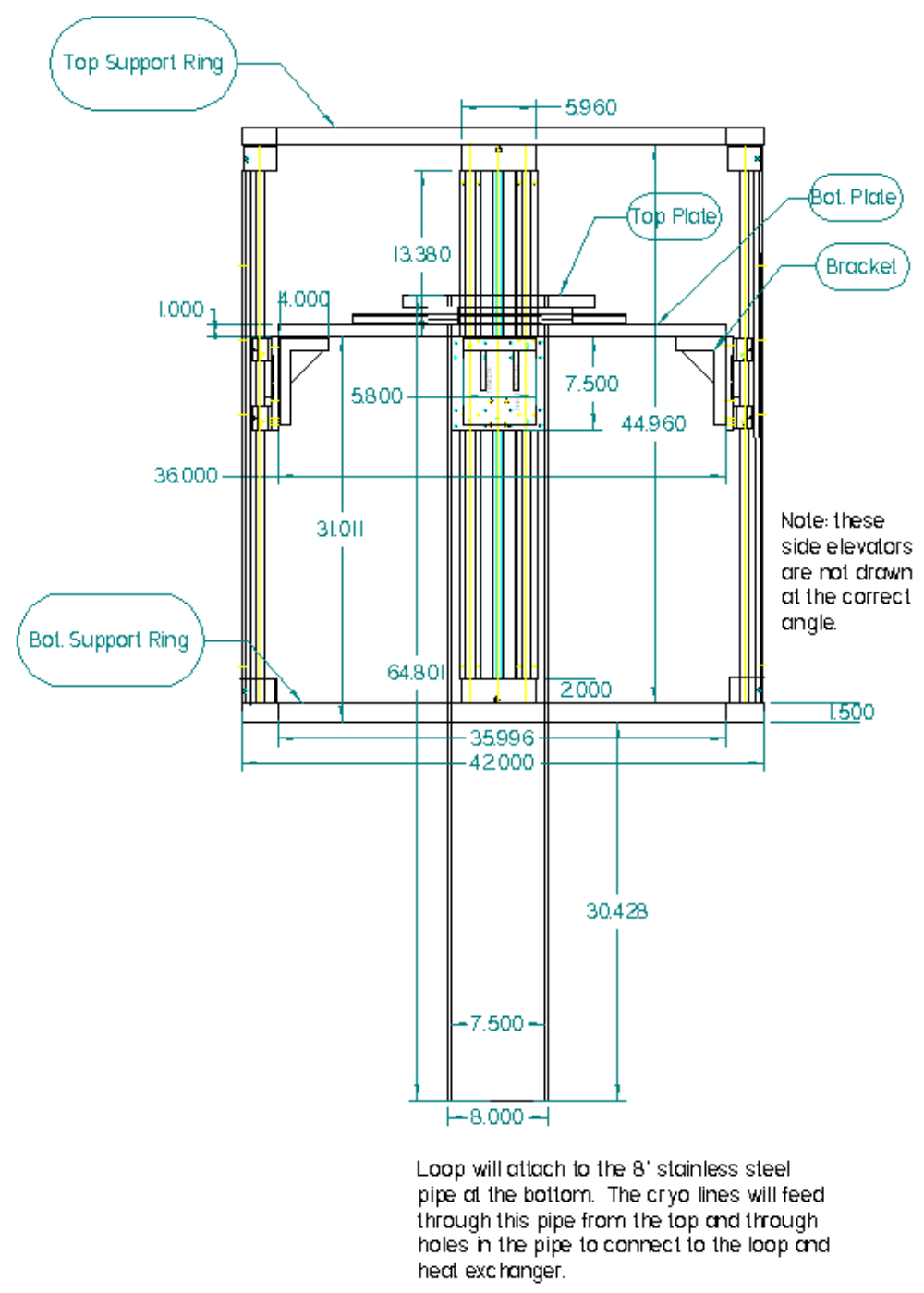

Figure 15: A side view of the rough drawing of the major elements of the motion mechanism.

out of the middle section. Two Thomson profile rail linear guides will be attached to the top of the circular plate. Each rail will have two pillow blocks or carriages. The rails will be Thomson 511 Style C Size 15 or 20 profile rails. There will be a square stainless steel plate (called the top plate in the figures) attached to the carriages. This plate will have a circular hole cut out of it and a 8" OD stainless steel pipe will be welded to the circular opening. The target loop will attach to the bottom of the 8" pipe. The length will be between 1 and 1.5 meters long to thermally isolate the target from the motion mechanism. A linear actuator (not shown in the figures) will sit on the bottom plate and drive the top plate to achieve horizontal target motion. In the current drawing, the motion could be as much as 4" from the center position. This is much more than the experiment will need for beam and target studies, but several inches of motion could be useful for initial positioning of the target. The linear actuator for the horizontal motion will not be used often; probably only in the beginning of the run for beam/target studies. After that, it will be de-energized and held in place by a brake. Since it is in a vacuum environment, special considerations are 
needed with this actuator. Since it is moving the target horizontally and for small distances, the acceleration and load will be small. Therefore, the actuator does not have to be very powerful. Heat transfer from the actuator may be a problem and we may have to thermal short it with the chamber wall.

\subsubsection{Vertical Travel}

The current thought is that the experiment requires three targets, a $35 \mathrm{~cm}$ liquid hydrogen cryotarget, a $35 \mathrm{~cm}$ aluminum dummy target, and a central carbon foil. In addition, there needs to be a target out position. A possible target configuration is shown in Figure 16. We can use the sketch and the maximum scattering angle $\left(\theta=14^{\circ}\right)$ to get an idea of the amount of vertical travel needed. One can see from the drawing that electrons that scatter from the front window of the cryotarget at 14 will be at least 1" from the dummy target. Also, the electrons scattered from the carbon target are free of interference. The "no target" position will be provided by rising the target above the beam line by a couple of centimeters. Hence, 12 " of vertical travel is sufficient for this target configuration.

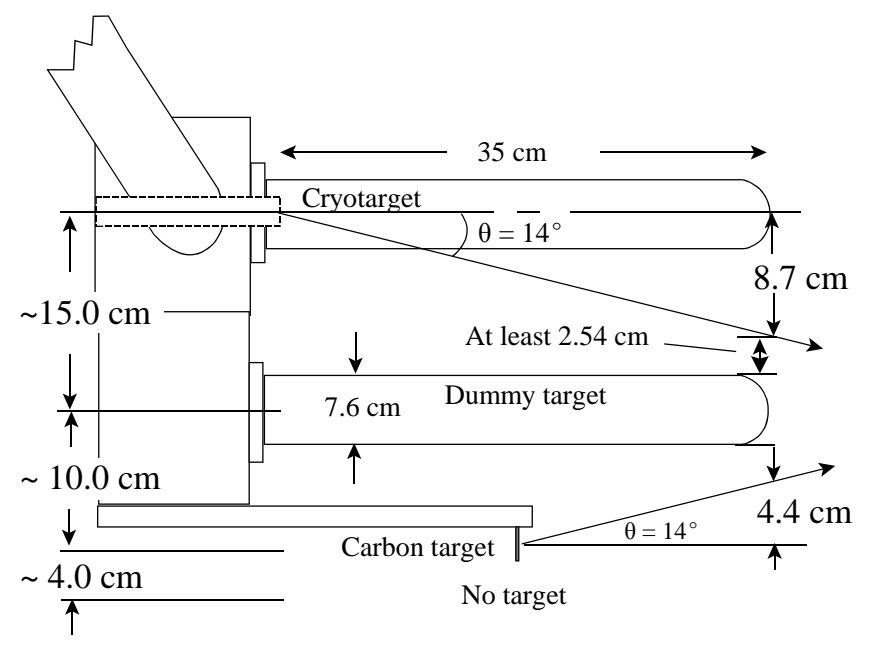

Figure 16: Possible configuration of the three targets

\subsubsection{Alignment}

The elevators will be aligned and made plumb on the floor before placing the motion mechanism in the chamber. The loop can also be attached to the 8" pipe on the floor. We will use a similar mechanism to attach the loop to the 8" pipe that is used for the standard Hall C cryotarget. This mechanism allows one to adjust the pitch, yaw, $x$, and $z$. We can simplify the design since we only need pitch, yaw, and $z$ adjustments. The loop will be made as symmetrically as possible to minimize cryogenic motion and since there is no vacuum load, we will not have vacuum motion. View ports will be used to determine the cryogenic motion and the $y$ and $x$ motions will be corrected by the motion mechanism. Repeatable $z$ cryogenic motion (along the beamline) can be corrected before closing up the chamber once the amount has been measured. 


\subsubsection{Heat Load}

It is important that the motion mechanism be thermally isolated from the cryotarget. The motion mechanism needs to be near room temperature and we need to minimize the heat load to the target. The heat conducted from the motion mechanism at $T_{2}=300 \mathrm{~K}$ to the cryotarget $T_{1}=20 \mathrm{~K}$ is given by $\dot{Q}=\frac{A}{l} \int_{T_{2}}^{T_{1}} K d T$ where $K$ is the thermal conductivity, $A$ is the cross sectional area of the 8 " stainless steel pipe and $l$ is the length. Stainless steel has a thermal integral from $300 \mathrm{~K}$ to $0 \mathrm{~K}$ of about $30 \mathrm{~W} / \mathrm{cm}$. Note, that when the cold end is at temperatures of $20 \mathrm{~K}$ or below there is little difference in the integral between using that temperature and $0 \mathrm{~K}$. For a length of $1 \mathrm{~m}(1.5 \mathrm{~m})$, the heat transfer is only about $12 \mathrm{~W}(8$ W) for the 8" (1/4" thick) stainless steel pipe. This is a small heat load to the target, but to ensure that the motion elements stay warm, we will place a temperature sensor at the top of the pipe and, if needed, a heating strap around the pipe.

In addition to producing the conceptual design for the motion mechanism, the MSU group designed and tested a failsafe way of operating the actual motor in the linear actuator that we altimately used for the target. This design allowed the controller to run the motor only if the brake is released and to engage the brake if the controller has any fault condition (which would remove the holding torque.) This capability was critical, since we cannot have the target drop if there was an amplifier fault and we cannot allow the motor to run if the brake was engaged. We also programmed the controller to run to the target positions and home, while also providing redundant protection via software limits and interlocks. Unfortunately, this controller is considered obsolete by the vendor and is very expensive. Hence, we did not have many spares. Therefore, we purchased a newer controller and tested it to ensure that it is a viable replacement for the current Qweak motion controller.

\subsection{Areal Densities for Hall C Experiments during 2005 running}

The PI is the target liason for the HMS experiments ${ }^{\ddagger}$ scheduled to run in late spring through mid-summer in Hall C. The experiments require similar solid targets to the ones that were used in run period starting in January, 2005. MSU graduate student, P. Junnarkar, remeasured the areal densities of these targets in the summer of 2005, but one of the carbon targets differed by $1 \%$ with the original measurement (exceeded the quoted error by a factor of 5 ). In addition, this carbon target was made up of two foils stacked together. The two foils were cut out of the same sample, yet their areal densities differed by $0.7 \%$. If these were accurate measurements, then this indicated that the foil's thickness was not as uniform as we thought. The areal density is determined from the ratio of mass and the area, which we can measure accurately, giving us the average areal density. Depending on the material, we then use a micrometer to measure the thickness (gives us an idea of the uniformity). The PI remeasured the two carbon foils, but instead of just measuring the coordinates of the corners as was done before, he measured the coordinates of the perimeter every $\mathrm{mm}$ (foils are approximately $25 \mathrm{~mm} \times 25 \mathrm{~mm}$ ). It turns out that these foils were not machined, and had wavy edges. From the perimeter coordinates, a more accurate area was determined and now

${ }^{\ddagger} \mathrm{E} 04-001: R=\sigma_{L} / \sigma_{T}$ in $A$ up to $Q^{2}=4 \mathrm{GeV}^{2}$; E06-009: $R=\sigma_{L} / \sigma_{T}$ in $D$ up to $Q^{2}=4 \mathrm{GeV}^{2}$; E05-017: $2 \gamma$ Exchange in $e p$ Rosenbluth Separation 
the two foils agree to better than $0.15 \%$. The overall or combined density remained about the same. Hence, we found a $1 \%$ error in the carbon density and resolved an issue with the apparent nonuniformity of the target.

\subsection{Spin Structure of Nucleons}

After nearly 30 years of experimental and theoretical work, the study of the nucleon spin structure has entered a mature stage, reaching beyond the exploration of the properties of the polarized structure function in the scaling regime into the region of the Bjorken scaling variable $x$ near its unity upper limit. The modern description of nucleon structure is done in terms of eight distributions functions, three of which are leading twist and independent of the quark transverse momentum $\vec{k}_{\perp} ; q(x)$ - related to the unpolarized structure (SF) $F_{1} ; \Delta q(x)$ - related to the spin SF $g_{1}$; and transversity $\delta(x)$ or $h_{T}(x)$. While the others involve both longitudinal and transverse components $\left(g_{T}=g_{1}+g_{2}\right.$; Collins $h_{1 T}^{\perp}$; Sivers $f_{1 T}^{\perp} ; h_{1 L}^{\perp}$; and $\left.h_{1}^{\perp}\right)$. Only $F_{1}, g_{1}$, and $g_{T}$ can be investigated with inclusive measurements, all others require semi-inclusive experiments.

In order to directly access $g_{2}$, one needs the absolute cross section difference for scattering longitudinally polarized electrons on transversely polarized nucleons. However, from an experimental point of view, it is easier to measure the spin asymmetry $A_{2}$. In fact one measures the parallel $A_{\|}$and perpendicular $A_{\perp}$ asymmetries, which are related to the spin asymmetries $A_{1}$ and $A_{2}$.

\subsubsection{Spin Asymmetries on the Nucleon Experiment: SANE}

The SANE experiment was proposed for Hall-C [32] to measure the inclusive double spin asymmetries by scattering polarized electrons on polarized protons, in the range of Bjorken $x$ from 0.3 to 0.8 and the corresponding range of four-momentum transfer from 2.5 to 6.5 $\mathrm{GeV}^{2}$. As discussed in the original SANE proposal [32], at the time there were little data on $A_{\|}$, and particularly $A_{\perp}$ in the region of $\left(Q^{2}, x\right)$ kinematic plane for $x>0.6$. The importance of gaining complete knowledge of the nucleon spin structure grows with time. The need for precise spin structure function data, $g_{T}$ in particular, may be best illustrated by the fact that one of DOE's 2011 milestones of the Performance measures for Hadronic Physics is to measure the proton spin structure functions $g_{1}$ and $g_{2}$ over the kinematic range delimited by $0.2<x<0.6$ and $1<Q^{2}<5 \mathrm{GeV}^{2}$. the large $x$ region is fascinating because it provides a window on proton structure in a regime where the sea quarks have been stripped away. One may hope that simple models can be applied to these "naked protons," potentially leading to insights into QCD, complementary to those obtained a generation ago from Constituent Quark Model descriptions of the baryon mass spectrum. Nucleon spin asymmetries at large $x$ may not only yield clues about $S U(6)$ (spin-flavor) symmetry breaking in confinement QCD, but are essential for the determination of all but the first moment of the spin structure functions. These moments are the natural connection between experiment and Lattice QCD, since lattice calculations do not directly determine spin observables like $A_{1}^{p}$, but only the lowest several moments of the various polarized and unpolarized parton distributions functions (PDF's). 
The SANE experiment ran in 2008, polarized beams of about $6 \mathrm{GeV}$ and $4.8 \mathrm{GeV}$ were scattered off solid ammonia material placed in the JLab/UVa polarized target. The target polarizing field was aligned parallel to the beam and at $80^{\circ}$ to the left of the beam, to measure $A_{\|}$and near- $A_{\perp}$. Obstruction of the scattered electrons by the target magnet coils necessitates the $80^{\circ}$ alignment, which has only a minor effect in the optimal extraction of $A_{1}$ and $A_{2}$. The projected results are shown in Fig. 17

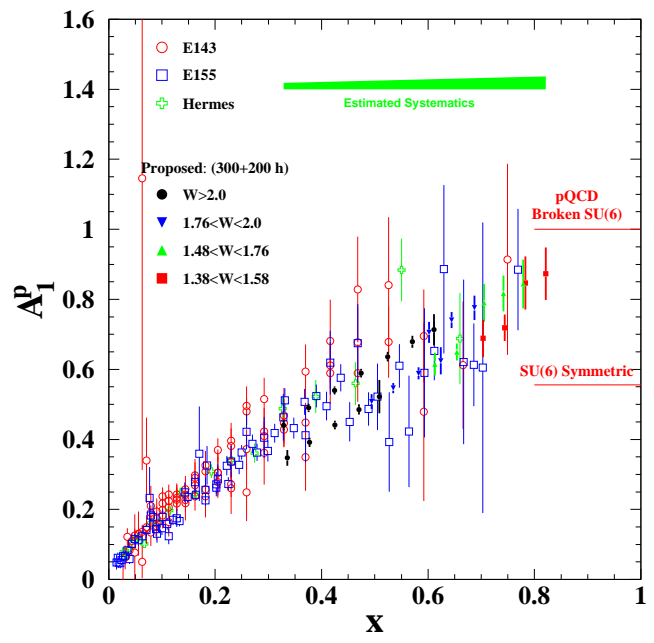

(a) World Data for $A_{1}^{p}$

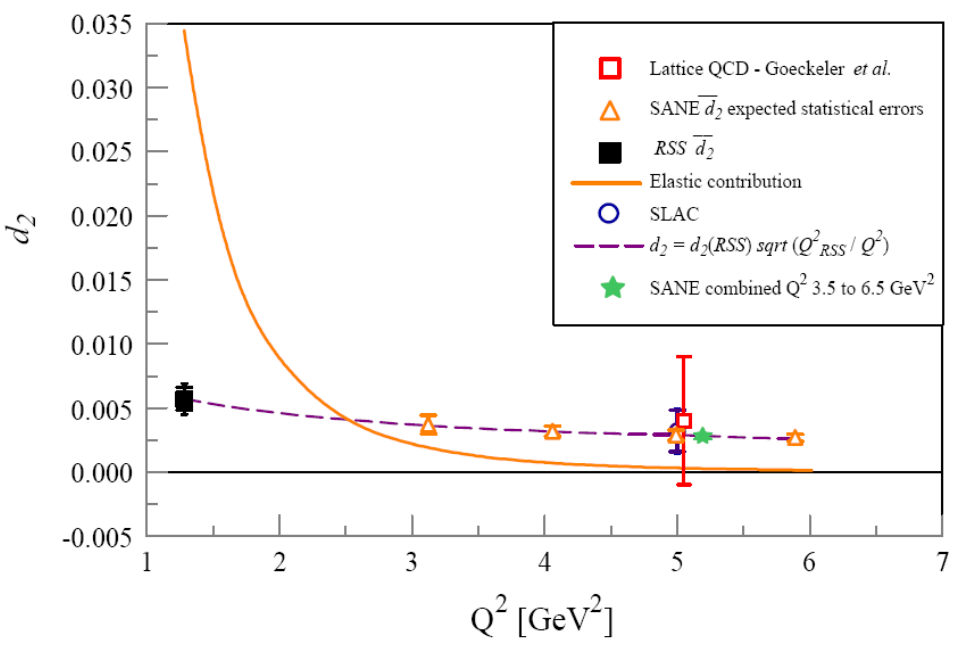

(b) $d_{2}$

Figure 17: (a) World's data for $A_{1}^{p}$ for high $x$ and SANE's projected uncertainties for both beam energies. The top locus of points is for $e=6 \mathrm{GeV}$ and the bottom locus is for $E=4.8$ $\mathrm{GeV}$. The $E=4.8 \mathrm{GeV}$ points have been shifted down for clarity. The two horizontal lines extending below $x=1$ represent the $S U(6)$ symmetric and pQCD predictions for $A_{1}^{p}$ at $x=1$. (b) Expected SANE $\bar{d}_{2}$ results for $5.7 \mathrm{GeV}$ beam energy (open triangles) plotted on top of $d_{2}\left(Q^{2}\right)=\bar{d}_{2}(R S S) \sqrt{Q^{2}(R S S) / Q^{2}}$ (dashed curve). The solid star represents the combination of the three highest $Q^{2}$ SANE points. The RSS (solid square) [30] with statistical and combined error bars, SLAC (open circle) [31] with total error, and lattice QCD (open square) results are also shown. The solid curve is the elastic contribution to $d_{2}$.

The PI and one of his graduate students, Luwani Ndukum, are actively participating in this experiment and Mr. Ndukum will write his PhD thesis from the analysis of this data. Prior to the running of SANE, the PI was the Beamline Manager for the polarized target experiments expected to run in Hall $\mathrm{C}$ during the fall 2008. It turned out that the main experiment, SANE E07-003, was the only experiment to run due to major target problems which caused more than a month downtime. Prior to the commissioning and running of the experiment, the beamline and target groups had meetings every two weeks (via conference calls and Virtual Network Connections (VNC)) to discuss the status and progress of their respective tasks. The major beamline elements that the PI worked on are listed below:

1. Upstream Beamline 
(a) Chicane Magnets in upstream beamline

i. Needed when running the transverse spin configuration of the target.

ii. Determined settings for the nominal beam energies.

iii. Coordinated with Hall C staff engineer, Bill Vulcan, to ensure each chicane magnet had appropriate power supply (which was an issue during last polarized target experiment).

(b) Coordinating with Machine Control Center (MCC) on polarized target girder.

i. Ensuring that MCC gets the proper signals from the beamline girder elements (i.e., SEM, BPMs, BCMs)

ii. Designed the radiator to be used with the Wide Angle Compton experiment that will use the polarized target.

2. Downstream Beamline

(a) Helium bag

i. Modified support of helium bag (e.g., added support tabs to prevent sagging); working with Hall $\mathrm{C}$ designers on support structure.

ii. Modified helium bag extension piece to minimize background.

iii. Determined placement of rear windows of the helium bag based on nominal beam energies during transverse running.

3. Responsible for the beamline sections safety documents for SANE.

(a) Experiment Safety Assessment Document

(b) Conduct of Operations Document

(c) Radiation Safety Assessment Document

\section{Publications and Reports}

- Ambrozewicz, P., et al., Near Threshold Electroproduction of the $\omega$ Meson at $Q^{2} \approx$ $0.5 \mathrm{GeV} / \mathrm{c}^{2}$. Phys. Rev. C 70, 035203 (2004).

- Armstrong, D.S. et al., Qweak: A Precision Measurement of the Proton's Weak Charge. Eur. Phys. J. A24 S02:155-158, 2005.

- Jones, M.K. et al., Proton $G_{E} / G_{M}$ from beam-target asymmetry. Phys. Rev. C74:035201, 2006.

- Navasardyan, T., et al., The Onset of Quark-Hadron Duality in Pion Electroproduction. Phys. Rev. Lett. 98:022001, 2007.

- Tvaskis, V., et al., Longitudinal-Transverse Separations of Structure Functions at Low $Q^{2}$ for Hydrogen and Deuterium. Phys. Rev. Lett 98:142301, 2007. 
- Tadevosyan, V., et al., Determination of the pion charge form factor for $Q^{2}=0.60-1.60$ $(\mathrm{GeV} / \mathrm{c})^{2}$. Phys. Rev. C75:055205, 2007.

- Wesselmann, F.R., et al., Proton spin structure in the resonance region. Phys. Rev. Lett 98:132003, 2007.

- Mkrtchyan, H., et al., Transverse momentum dependence of semi-inclusive pion production. Phys. Lett. B665:20-25, 2008.

- Huber, G.M.., et al., Charged Pion Form Factor between $Q^{2}=0.60$ and $2.45 \mathrm{GeV}^{2}$. II. Determination of, and results for, the Pion Form Factor. Phys. Rev. C78, 045203, 2008.

- Blok, H.P., et al., Charged Pion Form Factor between $Q^{2}=0.60$ and $2.45 \mathrm{GeV}^{2}$. I. Measurements of the Cross Section for the $1 \mathrm{H}(\mathrm{e}, \mathrm{e}+) \mathrm{n}$ Reaction. Phys. Rev. C78, 0452022008.

- Bosted, P., et al., Search for Sub-threshold Photoproduction of $J / \psi$ Mesons. Phys. Rev. C79:015209, 2009.

- Dalton, M.M., et al., Electroproduction of $\eta$ Mesons in the S11(1535) Resonance Region at High Momentum Transfer. Phys. Lett. C80, 015205, 2009.

- Villano, A.N., et al., Neutral Pion Electroproduction in the Resonance Region at High $Q^{2}$. Phys. Rev. C80, 035203, 2009.

- Malace, S.P., et al., Applications of quark-hadron duality in $F_{2}$ structure function. Phys. Rev. C80, 035207, 2009.

- Seely, J., et al., New measurements of the EMC effect in very light nuclei. Phys. Rev. Lett. 103, 202301, 2009.

- Slifer, K., et al., Probing Quark-gluon Interactions with Transverse Polarized Scattering. Phys. Rev. Lett. 105, 101601, 2010.

- Baker, W., Bosted, P., Dunne, J., New SOS Cerenkov. Technical report about the new Jefferson Lab Hall C SOS high pressure cerenkov that was installed for the $J / \psi$ experiment.

- Dunne, J., Cryogenic target Motion Mechanism for the Qweak experiment. Technical report about the target motion system design for the Qweak experimente. 2005.

\section{Talks Presented at Professional Meetings}

- Invited to give presentation at XI International Conference on Hadron Sprctroscopy Hadron05 in Rio de Janeiro, Brazil in August, 2005 on subthreshold $J / \psi$ photoproduction. 
- Qweak Infastructure Target, invited talk, Jefferson Lab Hall C User Group Meeting, January 26, 2007.

- Subthreshold $J / \psi$ Production, invited talk, Jefferson Lab Hall C User Group Meeting, January 25, 2007.

- The Qweak Experiment A Search for New Physics at the TeV Scale by Measurement of the Protons Weak Charge Invited talk, MSU Arts \& Sciences Faculty Research Showcase, October 16, 2008.

\section{Personnel and Collaborators}

Faculty:

Graduate Students:

Undergraduate Students:

High School Student:
James A. Dunne (PI)

Adesh Subedi (2009 - present), Luwani Ndukum (2008 present), Craig Carrigee (2006 - 2008) MS degree in December, 2007, Parikshit Junnarkar (2004 - 2006) MS degree in December, 2006, Shayam Gadepalli (2005) Sean Duke (2010 - present), Garrett Dorman (2009 - 2011), Michael Frassetto (2009 - 2010), Daniel Brown (2007 - 2011), Chris Yancy (2006), David Pigg (2005 - 2006), Wesley Baker (2001 - 2004)

Khadijah Ransom (2005 - 2006)

- Adesh Subedi has worked with the PI shortly after starting at MSU on the $Q_{w}$ cryogenic target project. He has worked on several projects related to the cryogenic hydrogen target motion system and is currently stationed at the lab to support the operation of the target and to participate in the experiment. He has become one of the target experts and is the only Ph.D. student target expert. He is also working on the boiling studies and is tying up loose ends on the target performance in preparation for a NIM article. Lately, he has been working on the simulations and is doing an outstanding job.

- Luwani Ndukum started working with the PI during the summer of 2008. He participated in the SANE experiments by taking shifts and was trained as a target operator. He is currently working on the charge symmetric background primarily from $\pi_{0}$ decays. His thesis topic will focus on the low $\mathrm{x}$ transverse data.

- Craig Cariggee worked with the PI for two years. He worked on the $Q_{w}$ Monte Carlo and investigated whether or not using the transverse target cell would impact the experiment in a negative way. His studies showed that even with the added material, the rates at the detectors were not affected. He received a Master's degree in 2008.

- Parikshit Junnarkar received his Master's degree from the analysis of the Sub-threshold $J / \psi$ experiment. He worked the Monte Carlo for the SOS cerenkov. He studyed the performance for both electron and muon particle identification for the Sub-threshold $J / \psi$ experiment. He help develop the Monte Carlo for $J / \psi$ production. 
- Shayam Gadepalli is a Doctoral student, who started in Jan. 2005, started gearing up to work on the Qweak experiment, but then dropped out of program.

- Sean Duke built the spare high power heater for the Qweak cryogenic target.

- Daniel Brown built the original high power heater for the Qweak cryogenic target to would have been located in the conical transition piece of the downstream end of the heat exchanger. To reduce the volume of liquid hydrogen, these conical transition pieces were replaced with flanges. Therefore, an new heater needed to be designed and placed in a straight section of 3 inch pipe. Daniel helped design the new heater and machined the G10 boarded and built the heater.

- Wesley Baker has been working for me for two year and did significate work on the SOS cerenkov design during the summer of 2004.

- David Pigg is working on a gui and software for determining liquid hydrogen boiling from luminosity studies.

- Outreach Program: Khadijah Ransom is from the Mississippi School of Mathematics and Sciences and participated in reseach for the fall and spring semester. She also worked on liquid hydrogen boiling studies.

\subsection{Principal Collaborators in the Reporting Period}

College of William and Mary: D.S. Armstrong, T. Averett, K.A. Griffioen, R. Fersch, J.M. Finn

University of Connecticut: R. Jones, K. Joo

DAPNIA-SphN, Centre d'Etudes de Saclay, France: J.M. Laget

Instituto de Fisica, Mexico: J. Erler

California Institute of Technology: M. Ramsey-Musolf

Los Alamos National Laboratory: J.D. Bowman, G.S. Mitchell, S. Penttila, W.S. Wilburn

Louisiana Tech University: T.A. Forest, K. Johnston, N. Simicevic, S. Wells

University of Manitoba, Canada: J. Birchall, W. Falk, M. Gericke, L. Lee, S.A. Page, W.D. Ramsay, W.T.H. van Oers

Massachusetts Institute of Technology: T. Botto, K. Dow, M. Farkhondeh, W. Franklin, K. Grimm, S. Kowalski, Y. Prok, E. Tsentalovich

TJNAF: P. Bosted, A. Bruell, R. Carlini, S. Chattopadhyay, E. Chudakov, R. Ent, D. Gaskell, A. Lung, D.Mack, S. Majewski, D. Meekins, M. Poelker, J. Roche, G. Smith, S.A. Wood, C. Zorn

Virginia Polytechnic Institute: J. Mammei, R. Mammei, N. Morgan, M. Pitt, R. Suleiman University of Virginia: D. Crabb, D. Day, O. Rondon

TRIUMF, Canada: C.A. Davis, J. Doornbos, University of Massachusetts: R. Arnold, S. Rock, M. Wang

University of New Hampshire: S. Covrig, F.W. Hersman, M. Holtrop

Pennsylvania State University: M. Strikman

Yerevan Physics Institute, Armenia: A. Agalaryan, r. Asaturyan, H. Mkrtchyan, S. Stepanyan, V. Tadevosyan 
University of Northern British Columbia, Canada: E. Korkmaz, T. Porcelli Ohio University: A.K. Opper

Hampton University: M.E. Christy, C. Keppel

Dartmouth College :T. Smith

University of Winnipeg, Canada: J. Martin 


\section{Bibliography}

\section{References}

[1] G. Warren and O. Rondon, co-spokesperson, SANE Spin Asymmetries on the Nucleon Experiment, JLab Experiment E03-109.

[2] P. Bosted, D. Day, X. Jiang, and M. Jones, co-spokesperson, Semi-SANE Semi-Inclusive Spin Asymmetries on the Nucleon Experiment, JLab Experiment E04-1113.

[3] P. Bosted, X. Jiang, and F.R. Wesselmann, co-spokesperson, "A High Precision Measurement of the Deuteron Spin-Structure Function $g_{1}^{d} / F_{1}^{d}$, , JLab Experiment E07-011.

[4] SLAC E142, P.L. Anthony et al., Phys. Rev. Lett. 71, 959 (1993).

[5] Opportunities in Nuclear Science. A Long Range Plan for the Next Decade, Nuclear Science Advisory Committee, (2002). http://www.sc.doe.gov/henp/np/nsac/docs/LRP_5547_FINAL.pdf

[6] P. Bosted and J.A. Dunne, co-spokesperson, Sub-threshold $J / \psi$ Photoproduction, JLab Experiment E03-008. http://www.jlab.org/exp_prog/proposals/03/PR03-008.pdf

[7] E. Chudakov et al., JLAB-TN-01-007 (2001).

[8] S.J. Brodsky, E. Chudakov, P. Hoyer, and J.M. Laget. Phys. Lett. B498, 23 (2001).

[9] B. Gittelman et al., Phys. Rev. Lett.35,1616 (1975).

[10] U. Camerini, et al. Phys. Rev. Lett. 35, 483 (1975).

[11] R. Prepost, SLAC Summer Inst.1975:267 Lepton-Photon Symp.1975:24.

[12] L. Frankfurt and M. Strikman, Phys. Rev. D66, 031502 (2002).

[13] O. Benhar, S. Fantoni, and G.I. Lykasow, Eur. Phys. J. A5, 137 (1999).

[14] O. Benhar, A. Fabrocini, S. Fantoni, and I. Sick, Nucl. Phys. A579, 493 (1994).

[15] S.J. Brodsky, I. Schmidt, and G.F. de Teranond, Phys. Rev. Lett 64, 1011 (1990).

[16] R.L. Anderson et al. Phys. Rev. Lett. 38, 263 (1977).

[17] S.J. Brodsky and G.A. Miller, Phys. Lett. B412, 125 (1997).

[18] E.L. Berger and D. Jones, Phys. Rev D23, 1521 (1981).

[19] S.J. Brodsky and A.H. Mueller, Phys. Lett. B206, 685 (1988).

[20] B.Z. Zopeliovich and B.G. Zakharov, Phys. Rev D44, 3466 (1991).

[21] E. Chudakov, P. Bosted, and J.A. Dunne, et al., proposal to PAC-31, PR12-07-106, unpublished (2008). 
[22] S.C. Bennett and C.E. Wieman, Phys. Rev. Lett. 82, 2484, (1999); C.S Wood et al., Science 275, 1759 (1997).

[23] V.A. Dzuba and V.V. Flambaum, e-print physics/0005038.

[24] V.A. Dzuba, V.V. Flambaum, and J.S. Ginges, e-print hep-ph/0111019.

[25] NuTeV Collaboration: G.P. Zeller et al., Phys. Rev. Lett 88:091802 (2002).

[26] E-158 Collaboration: P.L. Anthony et al., Phys. Rev. Lett. 95:081601 (2005).

[27] D. Armstrong et al., Jefferson Lab Proposal E02-020 The $Q_{W}^{p}$ Experiment (2002); R. Carlini spokesperson.

[28] D.H. Beck and B.R. Holstein, Int. J. Mod. Phys. E10:1 (2001).

[29] M.J. Musolf et al., Phys. Rep. 239. 1 (1994).

[30] F.R. Wesselmann et al., [RSS Collaboration], nucl-ex/0608003.

[31] SLAC 143, K. Abe et al., Phys. Rev. D58 112003 (1998); SLAC E155, K. Abe et al., Phys. Lett. B458, 339 (1999); P. L Anthony et al., [E155 Collaboration], Physi. Lett. B458, 529 (1999); P. L. Anthony et al., [E155 Collaboration], Phys. Lett. b553, 18 (2003).

[32] S. Choi, Z. E. Mezzeani, O. Rondon-Aremayo, et al., JLab Proposal to PAC-30, E07-003, unpublished (2007). 